\title{
A VNS algorithm for the design of supplementary damping controllers for small-signal stability analysis
}

\author{
Elenilson de Vargas Fortes ${ }^{\mathrm{a}, *, 1}$, Leonardo H. Macedo ${ }^{\mathrm{b}}$, Percival Bueno de Araujo ${ }^{\mathrm{b}}$, Rubén Romero ${ }^{\mathrm{b}}$ \\ ${ }^{a}$ Goiás Federal Institute of Education, Science and Technology, Rua Maria Vieira Cunha, 775, Residencial Flamboyant, 75804-714 Jataí, GO, Brazil \\ ${ }^{\text {b }}$ ão Paulo State University, Avenida Brasil 56, Centro, PO Box 31, 15385-000 Ilha Solteira, SP, Brazil
}

\section{A R T I C L E I N F O}

\section{Article history:}

Received 5 April 2017

Received in revised form 7 June 2017

Accepted 12 June 2017

Available online 30 June 2017

\section{Keywords:}

Interline power flow controller

Power oscillation damping

Power system stabilizers

Variable neighborhood search algorithm

\begin{abstract}
A B S T R A C T
This paper presents a variable neighborhood search (VNS) optimization algorithm for the design of the parameters of supplementary damping controllers: power system stabilizers (PSS) and the interline power flow controller-power oscillation damping (IPFC-POD) set. The objective is to insert damping to local and inter-area oscillation modes in multimachine power systems. The electric power system dynamics is represented by the current sensitivity model. Simulations were carried out using three systems: a test system known as Two-Area Symmetrical and two real systems, the New England and the Reduced Southern Brazilian. The VNS method was compared with a multi-start algorithm in terms of performance, showing better convergence rates. The proposal was also able to obtain solutions with high damping levels, that showed to be robust when changes on the operating point of the power system were considered. Finally, it has been verified that the PSS controllers were effective for damping the local mode oscillations, while the IPFC-POD set operated mainly damping the inter-area modes.
\end{abstract}

(c) 2017 Elsevier Ltd. All rights reserved.

\section{Introduction}

Stability studies can be divided into two distinct branches: transient and dynamic stability. Transient stability deals with the first oscillations that occur in the system after a large disturbance, such as short circuits in the transmission network or loss of a generating unit. On the other hand, the dynamic stability, or small-signal stability, the focus of this work, is limited to the restoration of the

Abbreviations: AVR, automatic voltage regulator; BFO, bacterial foraging optimization; CSM, current sensitivity model; DMC, decentralized modal control; FACTS, flexible AC transmission system; GA, genetic algorithm; GSA, gravitational search algorithm; hBFOA, hybrid bacteria foraging optimization algorithm; hPSO hybrid particle swarm optimization; IPFC, interline power flow controller; MFOA modified fruit fly optimization algorithm; PI, proportional-integral; POD, power oscillation damping; PSO, particle swarm optimization; PSS, power system stabilizer; SA, simulated annealing; SCBGA, specialized Chu-Beasley's genetic algorithm; SOA, seeker optimization algorithm; SSSC, static synchronous series compensators; SVC, static var compensator; TCSC, thyristor controlled series compensator; UPFC, unified power flow controller; VNS, variable neighborhood search; VSC, voltage source converter.

* Corresponding author.

E-mail addresses: elenilson.fortes@ifg.edu.br (E.V. Fortes), leohfmp@ieee.org (L.H. Macedo), percival@dee.feis.unesp.br (P.B.d. Araujo), ruben@dee.feis.unesp.br (R. Romero).

1 Principal corresponding author. steady state operation of the system after the occurrence of small disturbances that are often caused by small variations of loads [1].

In the literature, the problems of electromechanical oscillations are treated in different ways and can be of a local or global nature. Local problems involve a small part of the power system, and they are usually associated with the rotor angle oscillations of a generating unit and the rest of the system or another generating unit within its area. These oscillations are defined as local mode oscillations, and their frequencies of occurrence are within the range of $0.8-2.0 \mathrm{~Hz}[2]$.

The global problems are caused by interactions among a large number of generating units and basically involve a group of generators located in an area oscillating coherently against another group of generators, located on adjacent areas. These oscillations are referred to as inter-area modes, with oscillation frequencies in the range of $0.2-0.7 \mathrm{~Hz}$, occurring particularly when the systems are connected by relatively weak lines, i.e., lines with much lower capacity than the capacity of the systems that they interconnect [2,3].

To insert damping to these oscillations present in the electric power system, control strategies should be applied. These strategies are based on two types of controllers, known in the literature as the power system stabilizer (PSS) $[2,4]$ and power oscillation damping (POD) [5]. The PSSs are added to the control loop of the automatic voltage regulator (AVR) of synchronous generators and 


\section{Nomenclature}

$\Delta \omega_{k} \quad$ variation in the angular velocity of the rotor of generator $k$

$\Delta E_{k}^{f d} \quad$ field voltage of generator $k$

$\Delta P_{k m} \quad$ deviation in the active power of branch $\mathrm{km}$

$\Delta V_{k} \quad$ magnitude of the terminal voltage of the generator $k$

$\Delta V_{1_{k}}, \Delta V_{2_{k}}$ intermediary signals of the PSS dynamic model

$\Delta V_{j}^{p} \quad$ output modulated by the POD

$\Delta V_{k}^{r e f} \quad$ reference voltage of the AVR at generator $k$

$\Delta V_{1}^{\text {sup }} \quad$ output signal from the PSS

$\Delta V_{2}^{\text {sup }} \quad$ output signal from the POD

$\Delta Y_{1}, \Delta Y_{2}, \Delta Y_{3}$ intermediary signals of the POD's dynamic model

$\Delta X^{r e f}$ input signal of the POD

$\lambda_{i} \quad$ dominant eigenvalue $i$

$\sigma_{i} \quad$ real part of $\lambda_{i}$

$\omega_{i} \quad$ imaginary part of $\lambda_{i}$

$\omega_{n_{i}} \quad$ natural undamped frequency related to eigenvalue $i$

$\theta_{i} \quad$ voltage angle at bus $i$

$\xi_{i} \quad$ damping of eigenvalue $i$

$\xi_{i} \quad$ minimum damping for eigenvalue $i$

$\bar{b}_{i j}, r_{i j}, x_{i j}$ susceptance, resistance, and reactance of branch $i j$

$b_{i j}^{s h} \quad$ shunt susceptance of branch $i j$

$D_{k} \quad$ damping torque coefficient of the electromechanical loop of generator $k$

$H_{k} \quad$ inertia constant of generator $k$

$\mathbf{I}_{i j} \quad$ current phasor on branch $i j$

$I_{i}^{i n j, r}, I_{i}^{i n j, m}$ real and imaginary components of the current injection at bus $i$ by the IPFC

$I_{i j}^{r}, I_{i j}^{m} \quad$ real and imaginary components of $\mathbf{I}_{i j}$

$I_{i}^{r}, I_{i}^{m} \quad$ real and imaginary components of the current injection at bus $i$

$K_{1}^{p i}, K_{2}^{p i}, K_{3}^{p i}$ gains of the PI controllers

$K^{\text {pod }}$ gain of the POD
$K^{\text {pss }} \quad$ gain of the PSS

$K_{k}^{r} \quad$ gain of the AVR of generator $k$

$P_{k i}, Q_{k i}$ active and reactive power flows on branch $k i$

$P_{i}, Q_{i} \quad$ active and reactive power injections at bus $i$

$P_{i j}^{c}, Q_{i j}^{c} \quad$ active and reactive controlled power flows on branch $i j$

$P_{j}^{g}, Q_{j}^{g} \quad$ active and reactive power generations at bus $j$

$P_{i}^{\text {inj }} \quad$ active power injected at bus $i$ by the IPFC

$P_{j}^{l}, Q_{j}^{l} \quad$ active and reactive power demands at bus $j$

$P_{i j}^{r e f}, Q_{i j}^{r e f}$ active and reactive reference power flows on branch $i j$

$T_{d_{0 k}}^{\prime} \quad$ transient direct axis open-circuit time constant of gen-

$T_{1}^{p i}, T_{2}^{p i}, T_{3}^{p i}$

time constants of the PI controllers

$T_{\omega}^{\text {pod }} \quad$ time constant of the washout block of the POD model

$T_{\omega}^{p s s} \quad$ time constant of the washout block of the PSS model

$T_{1}^{p s s}, T_{2}^{p s s}, T_{3}^{p s s}, T_{4}^{p s s}$ time constants of the PSS

$T_{m}^{i p f c} \quad$ inherent delay of the control system of the IPFC

$T_{1}^{\text {pod }}, T_{2}^{\text {pod }}, T_{3}^{\text {pod }}, T_{4}^{\text {pod }}$ time constants of the IPFC-POD set

$T_{k}^{r} \quad$ time constant of the AVR of generator $k$

$V_{i} \quad$ voltage magnitude at bus $i$

$\mathbf{V}_{k}^{\prime} \quad$ complex voltage on the fictitious bus $k^{\prime}$

$V_{n}^{q}, V_{n}^{p} \quad$ direct and quadrature components of the series voltage

$\dot{V}_{n}^{p}, \dot{V}_{n}^{q} \quad \begin{aligned} & \text { source at bus } n \\ & \text { time derivatives of } V_{n}^{p} \text { and } V_{n}^{q}\end{aligned}$

$\mathbf{V}_{n}^{p q} \quad$ series synchronous controllable voltage source at bus $n$

$V^{\text {sup }} \quad$ output signal of the POD controller

$X_{1}, X_{2}, X_{3}$ input signals of the PI controllers

$\dot{X} 1 \dot{X}_{2}, \dot{X}_{3}$ time derivatives of $X_{1}, X_{2}$, and $X_{3}$

$x_{d_{k}}, x_{q_{k}}$ direct and quadrature axis synchronous reactances of

generator $k$

$x_{d_{k}}^{\prime} \quad$ direct axis transient reactance of generator $k$ operate inserting damping to local mode oscillations, if their control parameters (time constants and gains) are adjusted correctly $[3,6]$. The POD device can be used to increase the stability limits related to inter-area modes, if the device's control parameters (time constants and gain) are properly adjusted. The POD must also operate jointly with flexible AC transmission system (FACTS) devices. In this work, the FACTS interline power flow controller (IPFC) is considered [7].

Two types of analyzes will be performed: the static and the dynamic. Regarding the static analysis, the IPFC device is used to control, simultaneously or selectively, the power flow in certain transmission lines. Regarding the dynamic analysis, the structure of the IPFC's control system is modeled using proportional-integral (PI) controllers [3,6]. This model will be used to analyze the influence of the IPFC on small-signal stability in the electric power system.

To provide damping to unstable or low-damped modes of the electric power system and, thereby, increase its stability limits, a coordinated project of the supplementary controllers, PSS and IPFC-POD, is implemented. Among the existing methods in the literature, there are the classical methods and optimization techniques. The decentralized modal control (DMC), residue, and Nyquist's stability criterion are classified as classical methods [6,8-10].

The optimization algorithms are an alternative to the classical techniques for the design of these controllers. Since this design problem is nonlinear and has both algebraic and differential equations in the formulation, metaheuristics are suitable methods to be applied. In this context, the bacterial foraging optimization (BFO) algorithm is used in [11] to design the PSSs and in [12] to perform the coordinated design of PSSs and static var compensator (SVC) to improve the stability of the system. In [13], a particle swarm optimization (PSO) algorithm is presented to both allocate and set the parameters of SVCs and thyristor controlled series compensators (TCSC) to damp small-signal oscillations in multimachine power systems. Ref. [14] proposes a PSO algorithm to allocate and tune the parameters of PSSs and TCSCs to mitigate inter-area oscillations. Ref. [15] proposes a PSO algorithm for the coordinated design of PSS and TCSC in multimachine power systems. The genetic algorithm (GA) is used in [16] to perform the design of PSSs and unified power flow controller (UPFC) and in [17] to design PSSs and static synchronous series compensators (SSSC). In [18], the modified fruit fly optimization algorithm (MFOA) is used to perform the design of PSSs and SVC to improve small-signal stability in power systems with wind energy generation. The seeker optimization algorithm (SOA) is presented in [19] to coordinate PSSs and SSSC. A cuckoo search algorithm is proposed in [20] to design PSSs considering various operation conditions and disturbances in the power system. Ref. [21] presents a simulated annealing (SA) algorithm for the tuning of PSSs and the IPFC-POD.

Also, hybrid versions of optimization algorithms can be found, such as the hybrid bacteria foraging optimization-particle swarm optimization algorithm (hBFOA-PSO), that was used in [22] to perform the design of PSSs and SSSC, and in [23] for the design of TCSC. Finally, the hybrid particle swarm optimization-gravita tional search algorithm (hPSO-GSA) was presented in [24] for the coordinated design of PSSs and SSSC. 
In this study, a metaheuristic known as variable neighborhood search (VNS) [25] is used to perform the design of the parameters of the supplementary damping controllers, PSS and IPFC-POD. The VNS algorithm has shown excellent results in the field of Operations Research, but has not been explored in optimization problems of Electrical Engineering. The main advantage of the proposed technique is that it does not require defining and tuning parameters, such as the size of the population, mutation rate, etc., that are required, for example, in GAs. Most of the existing methods presented in the literature require tuning the algorithm to each instance of the problem, whereas the proposed VNS method can be applied, as it will be shown, to solve various instances of the problem without any modification. The proposed method has a local search strategy based on a cyclic coordinate method that uses a Fibonacci method to perform a line search. To validate the proposed technique, the performance of the VNS algorithm was compared to a multi-start algorithm [26]. Three test systems are considered: a small system known as Two-Area Symmetrical [1], and two real medium-sized systems, the New England [3] and the Reduced Southern Brazilian.

To represent the electric power system dynamics, the current sensitivity model (CSM) [3] was used, whose main concept is based on Kirchhoff's current law, which must be satisfied in all dynamic processes in the electric power system.

In this context, the main contributions of this work are as follows: (1) to implement computationally a VNS algorithm to perform the design of the PSS and IPFC-POD controllers; (2) to validate the VNS algorithm as an optimization technique for adjusting the parameters of the PSS and IPFC-POD controllers; and (3) to analyze the efficiency of the PSS and IPFC-POD controllers when the goal is to insert damping to local and interarea oscillation modes.

The remaining of the paper is organized as follows: Section 2 presents the model for the operation and control of the IPFC, while Section 3 shows the dynamic models for the PSS and POD controllers. Section 4 introduces the proposed VNS algorithm for the design of the PSS and POD controllers. Since the algorithms are presented in a general way in Section 4, Section 5 presents the details of the implementation of the optimization technique for the considered problem, based on the modeling presented in Sections 2 and 3. Section 6 presents the results of the work, including both static and dynamic analysis for the three systems, analysis of the performance of the proposed VNS algorithm, and a discussion about the results. Finally, Section 7 presents the conclusions and future work.

\section{Interline power flow controller}

The IPFC device can control power flows across lines, reducing overloads and compensating for voltage magnitude drops [7]. In Fig. 1, an equivalent circuit for the IPFC, with two voltage source converters (VSC), connected to the AC network via coupling transformers, is shown.
In Fig. 1, the IPFC is represented by two synchronous voltage sources injecting a series controllable voltage $\mathbf{V}_{n}^{p q}(n=j, k)$. Each voltage source is in series with its respective reactance $\left(x_{i n}, n=j, k\right)$ of the coupling transformer. The quantities $P_{i}$ and $Q_{i}$ are injections of active and reactive power in bus $i$. In addition, $P_{j i}, P_{k i}, Q_{j i}, Q_{k i}$, and $\mathbf{I}_{i j}, \mathbf{I}_{i k}, \mathbf{I}_{j i}$, and $\mathbf{I}_{k i}$ are, respectively, the active and reactive power flows and current phasors circulating on the lines between buses $j$ and $k$. The variables $\mathbf{V}_{j}^{\prime}$ and $\mathbf{V}_{k}^{\prime}$ are the voltages on the fictitious buses $j^{\prime}$ and $k^{\prime}$ and are included in the system for the deduction of the current injection model for the IPFC. Assuming that the active losses are zero, it follows that $\sum_{n=i, j, k} P_{n}^{i n j}=0$, i.e., the active power provided by one of the converters (VSC1) will always be equal to the active power demanded by the other (VSC2). This restriction is known as the active power invariance of the IPFC [27].

\subsection{Current injection model for the IPFC}

Eqs. (1)-(4) represent the current injection equations for the IPFC, presented in $[3,6]$, that can be obtained based on Fig. 1.

$$
\begin{aligned}
I_{i}^{r}= & -V_{i} \sin \theta_{i}\left(b_{i j}+b_{i k}\right)+b_{i j} V_{j} \cos \theta_{j}+b_{i k} V_{k} \sin \theta_{k} \\
& -\underbrace{\sum_{n=j, k} b_{i n}\left(V_{n}^{p} \cos \theta_{i}+V_{n}^{q} \sin \theta_{i}\right)}_{I_{i}^{i n j, r}} \\
I_{i}^{m}= & V_{i} \cos \theta_{i}\left(b_{i j}+b_{i k}\right)-\sum_{n=j, k} b_{i n} V_{n} \cos \theta_{n} \\
& +\underbrace{\sum_{n=j, k} b_{i n}\left(V_{n}^{q} \cos \theta_{i}-V_{n}^{p} \sin \theta_{i}\right)}_{I_{i}^{i n j, m}}
\end{aligned}
$$

$I_{n i}^{r}=b_{i n}\left(V_{i} \sin \theta_{i}-V_{n} \sin \theta_{n}\right)+\underbrace{b_{i n}\left(V_{n}^{p} \cos \theta_{i}+V_{n}^{q} \sin \theta_{i}\right)}_{I_{n}^{i n, r}}$

$I_{n i}^{m}=b_{i n}\left(V_{n} \cos \theta_{n}-V_{i} \cos \theta_{i}\right)+\underbrace{b_{i n}\left(V_{n}^{p} \sin \theta_{i}-V_{n}^{q} \cos \theta_{i}\right)}_{I_{n}^{i n, m}}$

In (1) $-(4), b_{\text {in }}=-\frac{1}{x_{i n}}$ for $n=j, k$. Additionally, $I_{i}^{r}$ and $I_{i}^{m}$ in (1) and (2) are the real and imaginary components of the current injection at bus $i$. Variables $I_{n}^{i n j, r}$ and $I_{n}^{i n j, m}$ in (3) and (4) are the current injections in bus $n(n=j, k)$ by the IPFC, the real and imaginary axis components, respectively.

\subsection{Structure of the IPFC control system}

With the current injection model for the IPFC presented, the next step is to derive a structure that represents the device's control system. PI controllers are used in the structure of the IPFC control system. Based on the fact that the quadrature components of the series voltage sources produce strong impact on active power flows and the in-phase components have significant effects on the reactive power flows, each controller shown in Fig. 2 is related

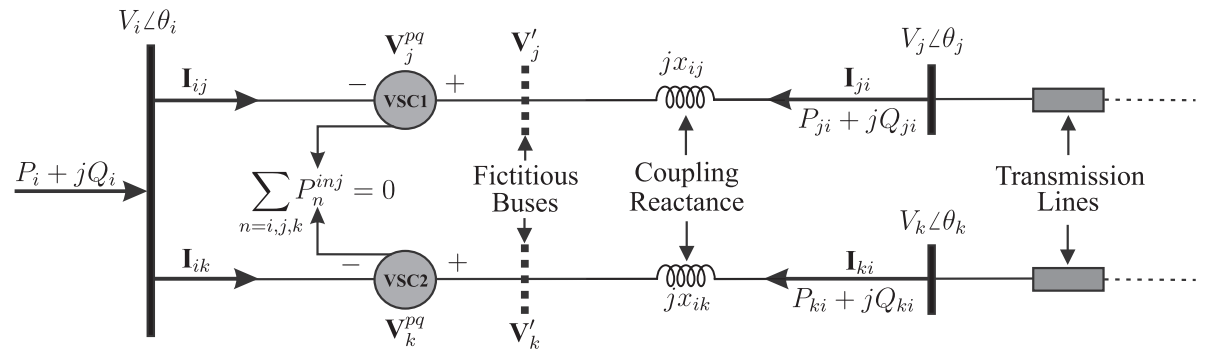

Fig. 1. Equivalent circuit of an IPFC with two series voltage sources. 
to a variable of the controllable sources $\left(V_{j}^{p}, V_{j}^{q}\right.$, and $\left.V_{k}^{p}\right)$, except the variable $V_{k}^{q}$, which is related to the equation of the invariance of the active power of the IPFC. The objective is to properly modulate, reducing the difference between the reference values and the measured values in the transmission lines, the active and reactive power flows that the IPFC device can control [3,6,28].

In the structure shown in Fig. 2, the controlled flows are $P_{i j}^{c}, P_{i k}^{c}$, and $Q_{i j}^{c}$, while the reference ones are $P_{i j}^{r e f}, Q_{i j}^{r e f}$, and $Q_{i k}^{r e f}$. The time constant $T_{m}^{i p f c}$ is the inherent delay of the control system and is in the range of 1-10 ms [2]. The parameters of the PI controllers are the gains $\left(K_{1}^{p i}, K_{2}^{p i}\right.$, and $\left.K_{3}^{p i}\right)$ and the time constants $\left(T_{1}^{p i}, T_{2}^{p i}\right.$, and $T_{3}^{p i}$ ). The signal $V^{\text {sup }}$ comes from the POD controller that, in this work, will be used to modulate the variable $V_{j}^{p}$ and, thus, provide damping to the oscillations of the electric power system. The signals $X_{1}, X_{2}$, and $X_{3}$ are input signals that are specified.

The dynamic behavior of the control structure shown in Fig. 2 for the IPFC device is given by (5)-(10), obtained by inspection.

$$
\begin{aligned}
& \dot{V}_{j}^{p}=\frac{K_{1}^{p i}}{T_{m}^{i p f c}}\left(P_{i j}^{r e f}-P_{i j}^{c}\right)+\frac{1}{T_{m}^{i f f c}}\left(X_{1}+V^{s u p}-V_{j}^{p}\right) \\
& \dot{X}_{1}=\frac{1}{T_{1}^{p i}}\left(P_{i j}^{r e f}-P_{i j}^{c}\right) \\
& \dot{V}_{j}^{q}=\frac{K_{2}^{p i}}{T_{m}^{i p f c}}\left(Q_{i j}^{r e f}-Q_{i j}^{c}\right)+\frac{1}{T_{m}^{i f f c}}\left(X_{2}-V_{j}^{q}\right) \\
& \dot{X}_{2}=\frac{1}{T_{2}^{p i}}\left(Q_{i j}^{r e f}-Q_{i j}^{c}\right)
\end{aligned}
$$

$$
\begin{aligned}
& \dot{V}_{k}^{p}=\frac{K_{3}^{p i}}{T_{m}^{i p f c}}\left(P_{i k}^{r e f}-P_{i k}^{c}\right)+\frac{1}{T_{m}^{i p f c}}\left(X_{3}-V_{k}^{p}\right) \\
& \dot{X}_{3}=\frac{1}{T_{3}^{p i}}\left(P_{i k}^{r e f}-P_{i k}^{c}\right)
\end{aligned}
$$

\section{Supplementary damping controllers PSS and POD}

This section presents the models of the PSS and IPFC-POD controllers used in this study. As shown in Fig. 3, the structures considered for both controllers are identical. They are represented by a gain ( $K^{\text {pss }}$ for the PSS and $K^{\text {pod }}$ for the POD), a washout block (with time constant $T_{\omega}^{p s s}$ for the PSS and $T_{\omega}^{p o d}$ for the POD), and two phase delay blocks (with time constants $T_{1}^{p S s}, T_{2}^{p s s}, T_{3}^{p s s}$, and $T_{4}^{p s s}$ for the PSS, and $T_{1}^{\text {pod }}, T_{2}^{\text {pod }}, T_{3}^{\text {pod }}$, and $T_{4}^{\text {pod }}$ for the POD). However, although they have the same structures, the operation mode and the input and output signals of each controller are distinct. The PSSs are coupled to the control loop of synchronous generators, whereas the POD is inserted in the control loop of FACTS devices (in this work, the IPFC).

Note that the input signal selected for the PSS is $\Delta \omega_{k}$ (variations in the angular velocity of the rotor of generator $k$ ), while for the POD, the adopted signal is $\Delta P_{k m}$ (deviations of the active power flow on the transmission line adjacent the IPFC-POD installation). The output of the PSS $\left(\Delta V_{1}^{\text {sup }}\right)$ is applied to the generator's voltage control loop, while the output of the POD $\left(\Delta V_{2}^{\text {sup }}\right)$ is added to the control loop of the IPFC device to modulate $\Delta V_{j}^{p}$.

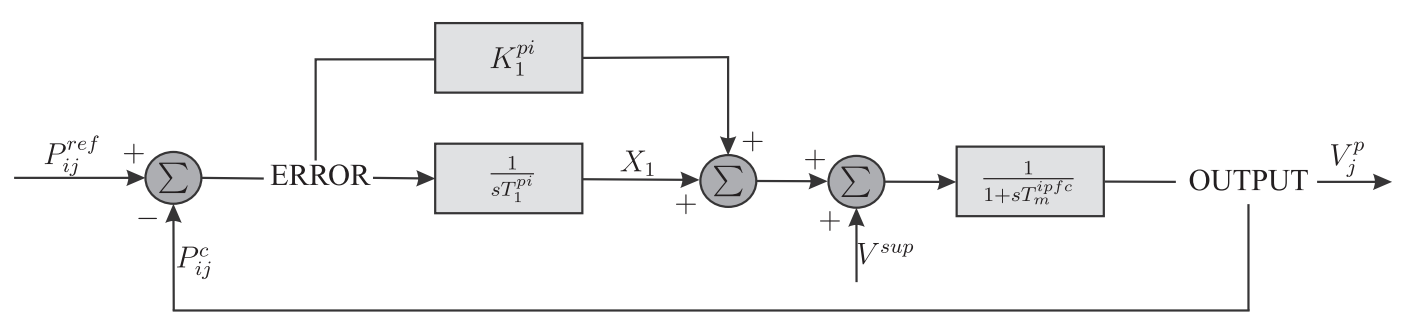

(a)

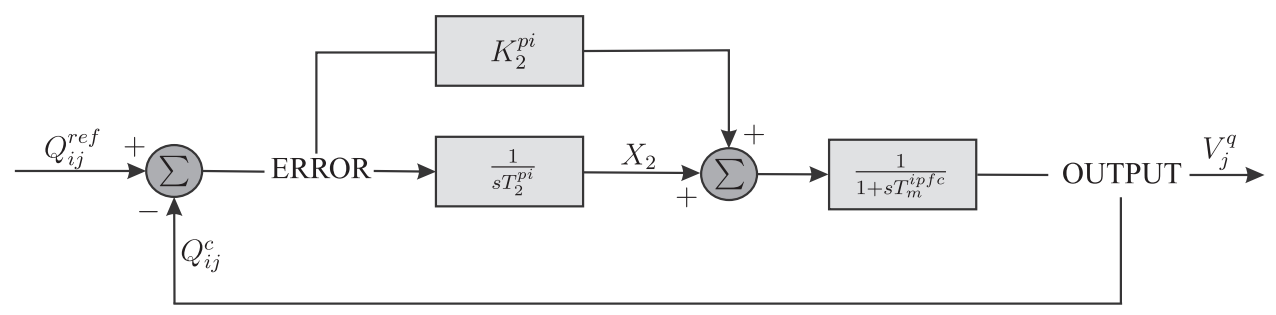

(b)

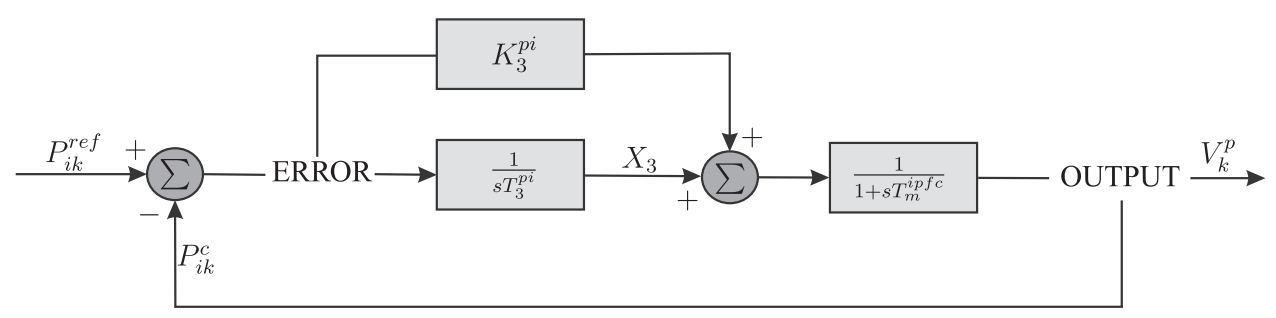

(c)

Fig. 2. Structure of the IPFC control system. 


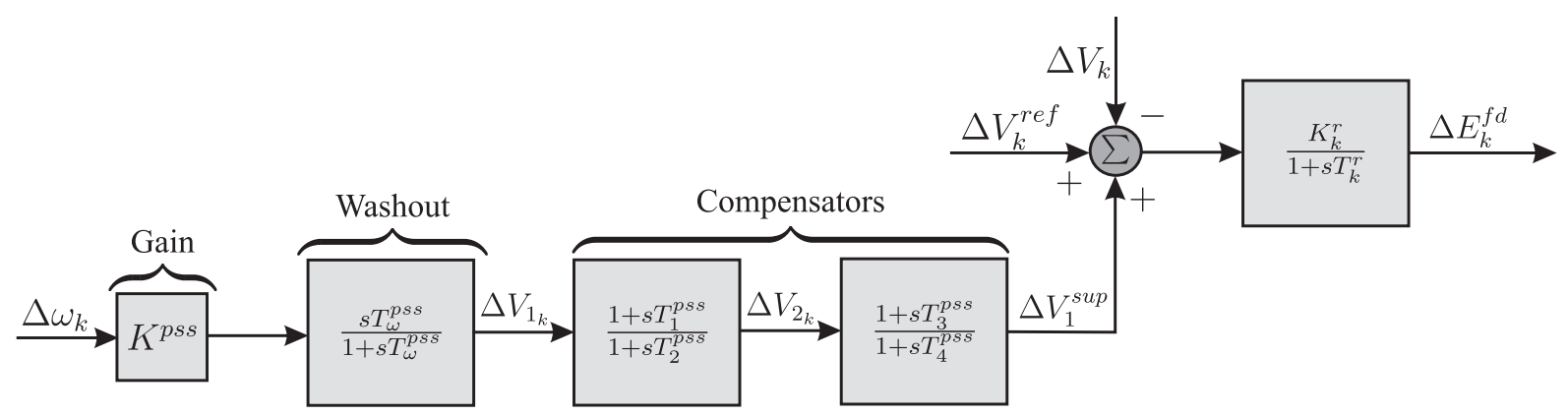

(a)

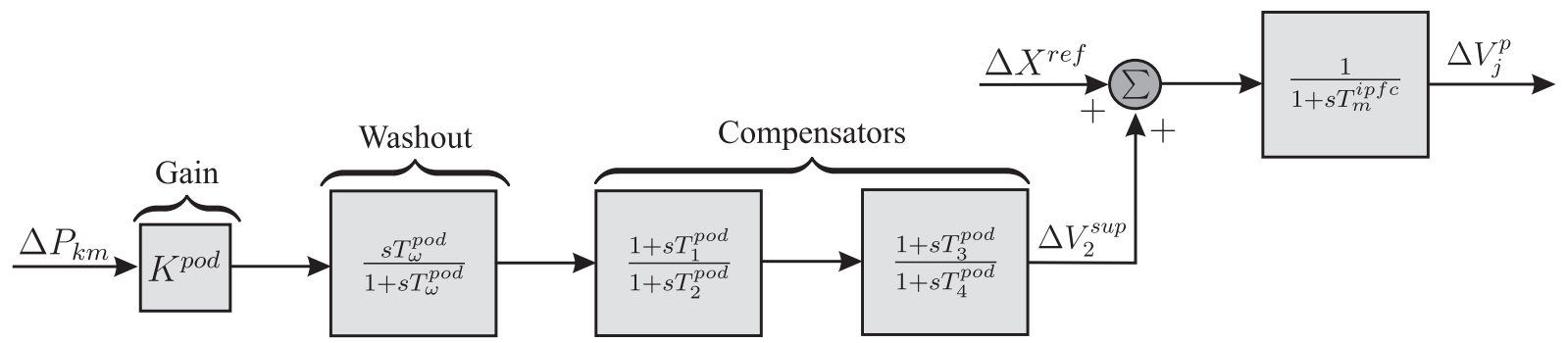

(b)

Fig. 3. Dynamic models of the (a) PSS controller, and (b) IPFC-POD controller.

In Fig. 3(a), the AVR is represented by a gain $K_{k}^{r}$ and a constant time $T_{k}^{r}$. The generator's field voltage is $\Delta E_{k}^{f d}$, the magnitude of the terminal voltage is $\Delta V_{k}$ and the reference voltage is $\Delta V_{k}^{\text {ref }}$. In Fig. 3 (b), $\Delta X^{\text {ref }}$ is the input signal and $T_{m}^{i p f c}$ is the time constant, both of them specified.

By analyzing the block diagrams shown in Fig. 3, the equations that define the dynamic behavior of the PSS controllers are shown in (11)-(14), while the dynamic operation of the POD controller is described by (15)-(18).

$$
\begin{aligned}
& \Delta \dot{V}_{1_{k}}=\Delta \dot{\omega}_{k} K^{\text {pss }}-\frac{1}{T_{\omega}^{p s s}} \Delta V_{1_{k}} \\
& \Delta \dot{V}_{2_{k}}=\frac{1}{T_{2}^{p s s}} \Delta V_{1_{k}}+\frac{T_{1}^{p s s}}{T_{2}^{p s s}} \Delta \dot{V}_{1_{k}}-\frac{1}{T_{2}^{p s s}} \Delta V_{2_{k}} \\
& \Delta \dot{V}_{1}^{\text {sup }}=\frac{1}{T_{4}^{p s s}} \Delta V_{2_{k}}+\frac{T_{3}^{p s s}}{T_{4}^{p s s}} \Delta \dot{V}_{2_{k}}-\frac{1}{T_{4}^{p s s}} \Delta V_{1}^{\text {sup }} \\
& \Delta \dot{E}_{k}^{f d}=\frac{K_{k}^{r}}{T_{k}^{r}}\left(\Delta V_{1}^{\text {sup }}+\Delta V_{k}^{r e f}-\Delta V_{k}\right)-\frac{1}{T_{k}^{r}} \Delta E_{k}^{f d} \\
& \Delta \dot{Y}_{1}=\frac{1}{T_{\omega}^{p o d}}\left(K^{\text {pod }} \Delta P_{k m}-\Delta Y_{1}\right) \\
& \Delta \dot{Y}_{2}=\frac{1}{T_{2}^{\text {pod }}}\left[\left(1-\frac{T_{1}^{p o d}}{T_{2}^{p o d}}\right)\left(K^{\text {pod }} \Delta P_{k m}-\Delta Y_{1}\right)-\Delta Y_{2}\right] \\
& \Delta \dot{Y}_{3}=\frac{1}{T_{4}^{p o d}}\left\{\left[\Delta Y_{2}+\frac{T_{1}^{p o d}}{T_{2}^{p o d}}\left(K^{\text {pod }} \Delta P_{k m}-\Delta Y_{1}\right)\right]\left(1-\frac{T_{3}^{p o d}}{T_{4}^{p o d}}\right)-\Delta Y_{3}\right\} \\
& \Delta \dot{V}_{j}^{p}=\frac{K_{1}}{T_{\omega}^{p o d}}\left(\Delta P_{j}^{r e f}-\Delta P_{j}^{c}\right)+\frac{1}{T_{\omega}^{p o d}}\left(\Delta X_{1}+\Delta V_{2}^{\text {sup }}-\Delta V_{j}^{p}\right)
\end{aligned}
$$

In (11)-(18), $\Delta V_{1_{k}}$ and $\Delta V_{2_{k}}$ are intermediary signals of the PSS dynamic model, while $\Delta Y_{1}, \Delta Y_{2}$, and $\Delta Y_{3}$ are intermediary signals of the POD dynamic model. More information about (11)-(18) can be found in $[3,29]$. Additionally, it is customary to adopt $T_{1}^{p s s}=T_{3}^{p s s}$ and $T_{2}^{p s s}=T_{4}^{p s s}$ for the PSS, and $T_{1}^{\text {pod }}=T_{3}^{\text {pod }}$ and $T_{2}^{\text {pod }}=T_{4}^{\text {pod }}$ for the POD [1].

\section{Technique for the design of the PSS and POD controllers}

This section presents the VNS algorithm proposed for the design of the parameters of PSS and POD controllers. The multi-start algorithm, used in the tests for comparison, is also discussed.

\subsection{VNS algorithm}

Existing exact methods for nonlinear optimization are only able to perform well when several requirements are satisfied in the problem, such as the continuity of the objective function and the constraints, the existence of the first and second derivatives of the objective function and constraints, and the convexity of the feasible region [30]. The problem addressed in this work, besides presenting nonlinear algebraic equations in the formulation, has differential equations that make it impossible to derive a complete exact mathematical model of optimization to be solved using these exact methods. For this reason, a modern heuristic approach for solving the presented problem is proposed in this section.

A local search strategy for nonlinear optimization is able to find a local optimum of a problem that presents many different good quality solutions, i.e., multi-modal problems. When exact optimization methods are applied directly to solve this type of problem, it is probable that only local optimal solutions, strongly dependent on the initial point, will be found. To overcome this limitation, Hansen and Mladenović presented in [25] a simple and efficient metaheuristic, called variable neighborhood search (Algorithm 1), which can be obtained by extending a local search algorithm to perform systematic changes of neighborhoods, for 
solving complex problems. Contrary to most local search techniques, the VNS algorithm does not follow a trajectory, but explores more distant neighborhoods of the current best solution.

According to Algorithm 1, initially, a sequence of neighborhoods $\mathcal{N}_{k}, k=1, \ldots, k_{\max }$ is defined. Since the problem in this work presents continuous variables, the neighborhoods are defined depending on the distance of a point $\mathbf{x}^{\prime}$ from the current solution $\mathbf{x}$. Thus, if a point $\mathbf{x}^{\prime}$ belongs to the hyperrectangle with center at $\mathbf{x}$, in which each edge $i$ has length $\varphi_{k}\left(\bar{x}_{i}-\underline{x}_{i}\right)$, where $\underline{x}_{i}$ and $\bar{x}_{i}$ are the lower and upper bounds of variable $i$, respectively, and $0<\varphi_{k} \leqslant 1$, $\varphi_{1}<\varphi_{2}<\cdots<\varphi_{k_{\max }}$, then $\mathbf{x}^{\prime} \in \mathcal{N}_{k}(\mathbf{x})$.

An initial solution $\mathbf{x}$, is then generated and its quality is evaluated using the objective function $F(\mathbf{x})$ presented in next section. In this work, this solution is randomly generated within the variables' bounds. The iterative process begins with $k \leftarrow 1$, and a solution $\mathbf{x}^{\prime}$ is randomly generated in the neighborhood $\mathcal{N}_{1}(\mathbf{x})$.

\section{Algorithm 1. Variable Neighborhood Search}

1. Initialization. Select the set of neighborhood structures $\mathcal{N}_{k}, k=1, \ldots, k_{\max }$, that will be used in the search; find an initial solution $\mathbf{x}$; choose a stopping condition;

2. Repeat the following until the stopping condition is met:

(a) Set $k \leftarrow 1$;

(b) Until $k=k_{\max }$, repeat the following steps:

i. Generate a point $\mathbf{x}^{\prime}$ at random from the $k^{\text {th }}$ neighborhood of $\mathbf{x}\left(\mathbf{x}^{\prime} \in \mathcal{N}_{k}(\mathbf{x})\right)$;

ii. Apply some local search method with $\mathbf{x}^{\prime}$ as initial solution; denote with $\mathbf{x}^{\prime \prime}$ the local optimum obtained; iii. If this local optimum is better than the best solution found in the process, move there $\left(\mathbf{x} \leftarrow \mathbf{x}^{\prime \prime}\right)$, and continue the search with $\mathcal{N}_{1}(k \leftarrow 1)$; otherwise, set $k \leftarrow k+1$, if $k>k_{\max }$, set $k \leftarrow 1$.

In the next step, a local search is applied with $\mathbf{x}^{\prime}$ as an initial solution. The local search used is presented in the next subsection. The solution of the local search is denoted $\mathbf{x}^{\prime \prime}$.

Three situations may occur:

- $\mathbf{x}^{\prime \prime}=\mathbf{x}$, i.e., $\mathbf{x}$ is the optimal solution of the neighborhood. In this case, $k \leftarrow k+1$, and the algorithm continues in the next neighborhood;

- $\mathbf{x}^{\prime \prime} \neq \mathbf{x}$ and $F\left(\mathbf{x}^{\prime \prime}\right) \geqslant F(\mathbf{x})$. In this case, a new local optimal was obtained, but with worse quality than the current solution. The algorithm continues in the next neighborhood $(k \leftarrow k+1)$;

- $\mathbf{x}^{\prime \prime} \neq \mathbf{x}$ and $F\left(\mathbf{x}^{\prime \prime}\right)<F(\mathbf{x})$. A new solution, better than the current solution, was found. The current solution is updated $\left(\mathbf{x} \leftarrow \mathbf{x}^{\prime \prime}\right)$, and the search restarts in the first neighborhood $(k \leftarrow 1)$ of $\mathbf{x}$.

If the last neighborhood is reached $\left(k>k_{\max }\right)$ and no improvement is obtained, then the search restarts in the first neighborhood $k \leftarrow 1$.

\subsection{Local search algorithm}

The local search step of the VNS algorithm is performed using the cyclic coordinate method [30] (Algorithm 2). This method can be directly used to optimize a nondifferentiable nonlinear function. A line search is repeatedly performed in the directions $\mathbf{d}_{1}, \ldots, \mathbf{d}_{n}$, where $\mathbf{d}_{i}$ is a vector in which element $i$ is a " 1 " and all the remaining elements are zeros, from an initial point $\mathbf{x}_{1}$. The order of the directions, in this paper, is generated randomly.
However, in order to perform a local search, only a fraction of the interval $\underline{x}_{i}$ and $\bar{x}_{i}$ of each variable is used, around the current solution of the VNS algorithm. The interval considered for each variable represented twenty percent of the total amplitude in each direction from the current solution $\mathbf{x}$, i.e., $x_{i}-\rho\left(\bar{x}_{i}-\underline{x}_{i}\right) \leqslant x_{i} \leqslant$ $x_{i}-\rho\left(\bar{x}_{i}-\underline{x}_{i}\right), \rho=0.2$. If a variable violates a limit $\underline{x}_{i}$ or $\bar{x}_{i}$, the value is fixed at the violated limit.

On the other hand, in order to validate this proposal, the cyclic coordinate method is applied directly to the problem in the multistart algorithm, considering the complete intervals for each variable.

\section{Algorithm 2. Cyclic Coordinate Method}

1. Initialization. Choose a scalar $\epsilon>0$ to be used for terminating the algorithm, and let $\mathbf{d}_{1}, \ldots, \mathbf{d}_{n}$ be the coordinated directions. Choose an initial point $\mathbf{x}_{1}$, let $\mathbf{y}_{1} \leftarrow \mathbf{x}_{1}$, let $k \leftarrow 1$ and $j \leftarrow 1$, and go to the Main Step;

\section{Main Step.}

(a) Let $\gamma_{j}$ be an optimal solution to the problem to minimize $F\left(\mathbf{y}_{j}+\gamma \mathbf{d}_{j}\right)$ subject to $\gamma \in\left[a_{1}, b_{1}\right]$ and let $\mathbf{y}_{j+1} \leftarrow \mathbf{y}_{j}+\gamma_{j} \mathbf{d}_{j}$. If $j<n$, replace $j$ by $j+1$, and repeat Step (a). Otherwise, if $j=n$, got to Step (b);

(b) Let $\mathbf{x}_{k+1} \leftarrow \mathbf{y}_{k+1}$. If $\left\|\mathbf{x}_{k+1}-\mathbf{x}_{k}\right\|<\epsilon$, then stop. Otherwise, let $\mathbf{y}_{1} \leftarrow \mathbf{x}_{k+1}$, let $j \leftarrow 1$, and $k \leftarrow k+1$, and go to Step (a).

The Fibonacci line search method [30] (Algorithm 3), which is a derivative-free algorithm, was chosen to minimize $F$ in a given direction $\mathbf{d}_{i}$. This method minimizes a strictly quasiconvex function in a bounded interval $\left[a_{1}, b_{1}\right]$. The Fibonacci sequence $\left\{\mathcal{F}_{k}\right\}$, defined as $\mathcal{F}_{k+1} \leftarrow \mathcal{F}_{k}+\mathcal{F}_{k-1}, k=1,2, \ldots$, with $\mathcal{F}_{0}=\mathcal{F}_{1}=1$, is used for reducing the interval of uncertainty.

\section{Algorithm 3. Fibonacci Line Search Method}

1. Initialization. Define a suitable final length of uncertainty $l>0$ and a constant $\epsilon>0$. Consider $\left[a_{1}, b_{1}\right]$ as the initial interval of uncertainty, and choose the number of calculations $n$ such that $\mathcal{F}_{n}>\left(b_{1}-a_{1}\right) / l$;

Let $\gamma_{1} \leftarrow a_{1}+\left(\mathcal{F}_{n-2} / \mathcal{F}_{n}\right)\left(b_{1}-a_{1}\right)$ and $\mu_{1} \leftarrow a_{1}+\left(\mathcal{F}_{n-1} / \mathcal{F}_{n}\right)\left(b_{1}-a_{1}\right)$. Evaluate $F\left(\mathbf{y}_{j}+\gamma_{1} \mathbf{d}_{j}\right)$ and $F\left(\mathbf{y}_{j}+\mu_{1} \mathbf{d}_{j}\right)$,

let $k \leftarrow 1$, and go to the Main Step;

\section{Main Step.}

(a) If $F\left(\mathbf{y}_{j}+\gamma_{k} \mathbf{d}_{j}\right)>F\left(\mathbf{y}_{j}+\mu_{k} \mathbf{d}_{j}\right)$, go to Step (b); and if $F\left(\mathbf{y}_{j}+\gamma_{k} \mathbf{d}_{j}\right) \leqslant F\left(\mathbf{y}_{j}+\mu_{k} \mathbf{d}_{j}\right)$, go to Step (c);

(b) Let $a_{k+1} \leftarrow \gamma_{k}$ and $b_{k+1} \leftarrow b_{k}$. Furthermore, let $\gamma_{k+1} \leftarrow \mu_{k}$, and let $\mu_{k+1} \leftarrow a_{k+1}+\left(\mathcal{F}_{n-k-1} / \mathcal{F}_{n-k}\right)\left(b_{k+1}-a_{k+1}\right)$.

If $k=n-2$, go to Step (e); otherwise, evaluate

$F\left(\mathbf{y}_{j}+\mu_{k+1} \mathbf{d}_{j}\right)$ and go to Step (d);

(c) Let $a_{k+1} \leftarrow a_{k}$ and $b_{k+1} \leftarrow \mu_{k}$. Furthermore, let $\mu_{k+1} \leftarrow \gamma_{k}$, and let $\gamma_{k+1} \leftarrow a_{k+1}+\left(\mathcal{F}_{n-k-2} / \mathcal{F}_{n-k}\right)\left(b_{k+1}-a_{k+1}\right)$.

If $k=n-2$, go to Step (e); otherwise, evaluate $F\left(\mathbf{y}_{j}+\gamma_{k+1} \mathbf{d}_{j}\right)$ and go to Step (d);

(d) Set $k \leftarrow k+1$ and go to Step (a);

(e) Let $\gamma_{n} \leftarrow \gamma_{n-1}$ and $\mu_{n} \leftarrow \gamma_{n-1}+\epsilon$. If

$F\left(\mathbf{y}_{j}+\gamma_{n} \mathbf{d}_{j}\right)>F\left(\mathbf{y}_{j}+\mu_{n} \mathbf{d}_{j}\right)$, let $a_{n} \leftarrow \gamma_{n}$ and $b_{n} \leftarrow b_{n-1}$.

Otherwise, if

$F\left(\mathbf{y}_{j}+\gamma_{n} \mathbf{d}_{j}\right) \leqslant F\left(\mathbf{y}_{j}+\mu_{n} \mathbf{d}_{j}\right)$, let $a_{n} \leftarrow a_{n-1}$ and $b_{n} \leftarrow \gamma_{n}$. Stop; the optimal solution is in the interval $\left[a_{n}, b_{n}\right]$. 


\subsection{Multi-start method}

The multi-start algorithm (Algorithm 4) is an optimization method based on a strategy for restarting a search strategy from many different solutions. Basically, the technique presented in this paper consists of two steps: generating a random solution and performing an improvement step. These two steps must be repeated until the stop criterion is satisfied. The search strategy used is the same one used in the VNS algorithm (considering, in this case, the complete intervals for each variable). The efficiency of the multi-start algorithm will be compared to the VNS algorithm, in order to evaluate the performance of the latter.

Algorithm 4. Multi-Start Algorithm

1. Initialization. Set $k \leftarrow 1$;

2. Main Step. Until $k=k_{\max }$, repeat the following steps:

(a) Generation. Construct solution $\mathbf{x}_{k}$;

(b) Search. Apply a search method to improve $\mathbf{x}_{k}$; Let $\mathbf{x}_{k}^{\prime}$ be the solution obtained;

(c) Update. If $\mathbf{x}_{k}^{\prime}$ improves the best solution, update it; set $k \leftarrow k+1$.

\section{Evaluation function and bounds for the optimization procedure}

Consider that $\mathbf{x}$ is a solution proposal obtained in the algorithms of the previous section. Its evaluation function, $F(\mathbf{x})$, is defined as shown in (19).

$F(\mathbf{x})=\sum_{i=1}^{n} \max \left\{\underline{\xi}_{i}-\xi_{i}, 0\right\}$

In (19), $n$ is the number of eigenvalues of interest in the problem, $\xi_{i}$ is the damping of eigenvalue $i$ in solution $\mathbf{x}$, and $\underline{\xi}_{i}$ is the minimum desired damping for eigenvalue $i$, defined by the system operator. Note that each $\xi_{i}$ is determined in the CSM.

The proposed evaluation function will assume a value of zero when each eigenvalue of interest $i$ presents a damping greater than $\xi_{i}$. Because it is a continuous optimization problem, it is assumed that convergence is achieved when $F(\mathbf{x})<\epsilon$, where $\epsilon=0.005$.

A solution proposal is illustrated in Fig. 4, which contains the control variables of the problem, in this case, the time constants and gains of the supplementary damping controllers, PSS and IPFC-POD.

In Fig. 4, it is considered the representation of a solution proposal for an electric power system equipped with $n$ PSSs and one IPFC-POD set, and $T_{1_{l}}^{p s s}, T_{2_{l}}^{p s s}, K_{l}^{p s s}$ and $T_{1}^{\text {pod }}, T_{2}^{\text {pod }}, K^{\text {pod }}$ are the time constants and gains of the PSS controllers and IPFC-POD set, respectively.

In all the simulations performed, it was considered that the time constants of the washout blocks of both controllers and the inherent delay of the control device are fixed: $T_{\omega}^{p s s}=10$ seconds for the PSS (Fig. 3(a)), $T_{\omega}^{\text {pod }}=1$ second and $T_{m}^{i p f c}=0.005$ seconds (Fig. 3(b)) for the IPFC-POD set. In addition, the values of the coupling reactances of the transformers are equal to 0.01 p.u. $\left(x_{i n}, n=j, k\right)$.

Each variable of a solution proposal is bounded by its respective limits, as indicated in Table 1.

Next section presents the results obtained with the proposed method.

\section{Tests and results}

Three systems were used in the simulations: the Two-Area Symmetrical system [1], the New England system [3], and the Reduced Southern Brazilian system. The first objective was to test and validate the VNS algorithm proposed in this paper. The second objective was to show that PSS controllers perform damping local oscillation modes, while the IPFC-POD inserts damping to the inter-area modes.

\subsection{Characteristics of the test systems}

The Two-Area Symmetrical system, shown in Fig. 5(a), has 2 areas, 4 generators, 17 branches, and 12 buses, including two new buses $\left(\mathrm{FB}_{1}^{k}\right.$ and $\left.\mathrm{FB}_{2}^{k}\right)$ and two new transmission lines $\left(7-\mathrm{FB}_{1}^{k}\right.$ and $\left.7-\mathrm{FB}_{2}^{k}\right)$, added in order to perform simulations with the IPFC. It was considered that this system was equipped with two PSSs, at generators G2 and G3, and an IPFC-POD (see Fig. 5(a)).

The New England system consists of 10 generators, 41 buses, and 48 transmission lines and its line diagram is shown in Fig. 5 (c). In this diagram, two fictitious buses, $\mathrm{FB}_{1}^{n}$ and $\mathrm{FB}_{2}^{n}$, and two lines, $37-\mathrm{FB}_{1}^{n}$ and $37-\mathrm{FB}_{2}^{n}$, were included to perform simulations with the IPFC. This system was equipped with eight PSSs, at generators G1, G2, G3, G4, G5, G7, G8, and G9, and an IPFC-POD (see Fig. 5(c)).

Fig. 6 shows the Reduced Southern Brazilian system. This is a real system that comprises 10 generators, 45 buses, and 73 transmission lines. It was equipped with nine PSSs at generators $G 1, G 2$, G3, G4, G6, G7, G8, G9, and G10 (see Fig. 6). The locations of the PSSs in all systems were determined by the participation factors [1], which indicated the generators that were more involved in the formation of each local mode.

\subsection{Static analysis}

The case in which the IPFC was installed in the system, but did not perform the control of active and reactive power flows, will be referred to as Case A. The other case, when the IPFC was actuating, will be referred to as Case B. Table 2 shows the values of the variables that were used to represent the IPFC's series converters in the systems in which they were installed.

In Case A, shown in Table 2, it can be seen that the values of the variables that were used to represent the series converters of the IPFC device are zero, which indicates that the IPFC was included in the system, but it was not performing any control.

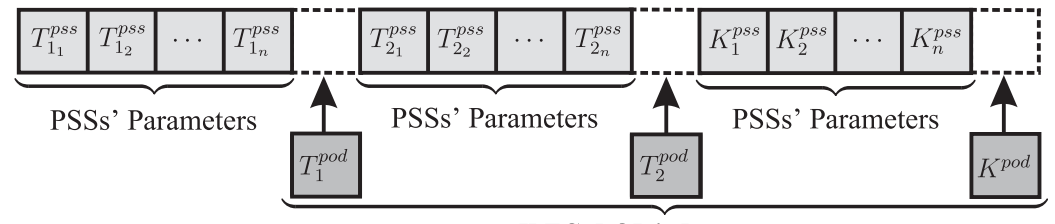

IPFC-POD's Parameters

Fig. 4. Representation of a solution proposal. 
Table 1

Bounds of the variables.

\begin{tabular}{|c|c|c|c|c|c|c|c|}
\hline System & Limits & $T_{1_{l}}^{p s s}[\mathrm{~s}]$ & $T_{2_{l}}^{p s s}[\mathrm{~s}]$ & $K_{l}^{p s s}$ [p.u.] & $T_{1}^{\text {pod }}[\mathrm{s}]$ & $T_{2}^{p o d}[\mathrm{~s}]$ & $K^{\text {pod }}$ [p.u.] \\
\hline \multirow[t]{2}{*}{ Two-Area Symmetrical } & Min. & 0.05 & 0.05 & 1.00 & 0.05 & 0.05 & 0.05 \\
\hline & Max. & 1.00 & 1.00 & 5.00 & 1.00 & 1.00 & 1.50 \\
\hline \multirow[t]{2}{*}{ New England } & Min. & 0.10 & 0.01 & 1.00 & 0.10 & 0.10 & 0.10 \\
\hline & Max. & 1.50 & 0.10 & 10.00 & 0.40 & 0.40 & 0.50 \\
\hline \multirow[t]{2}{*}{ Reduced Southern Brazilian } & Min. & 0.01 & 0.01 & 1.00 & - & - & - \\
\hline & Max. & 0.50 & 0.25 & 12.00 & - & - & - \\
\hline
\end{tabular}

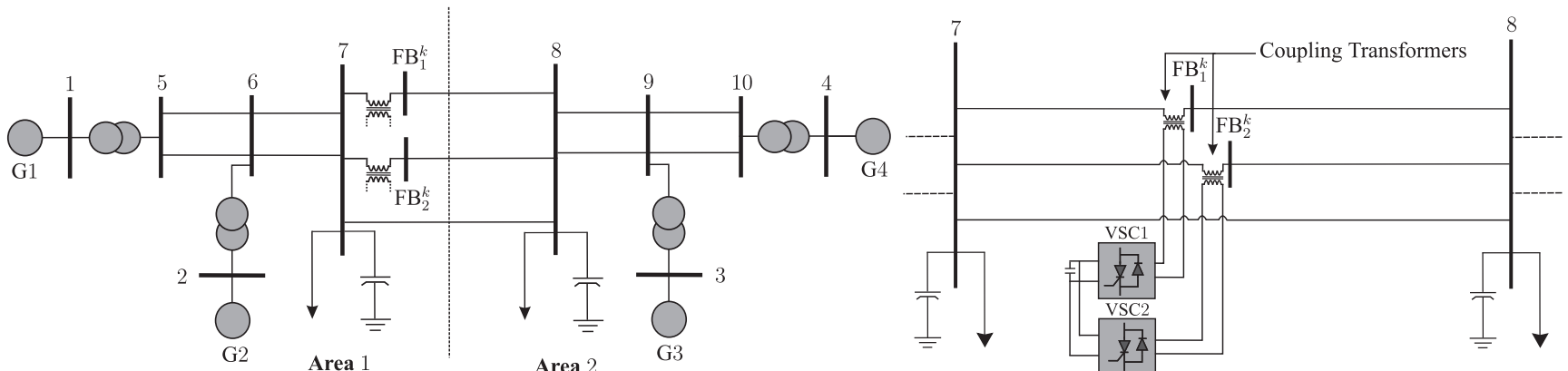

(a)

(b)

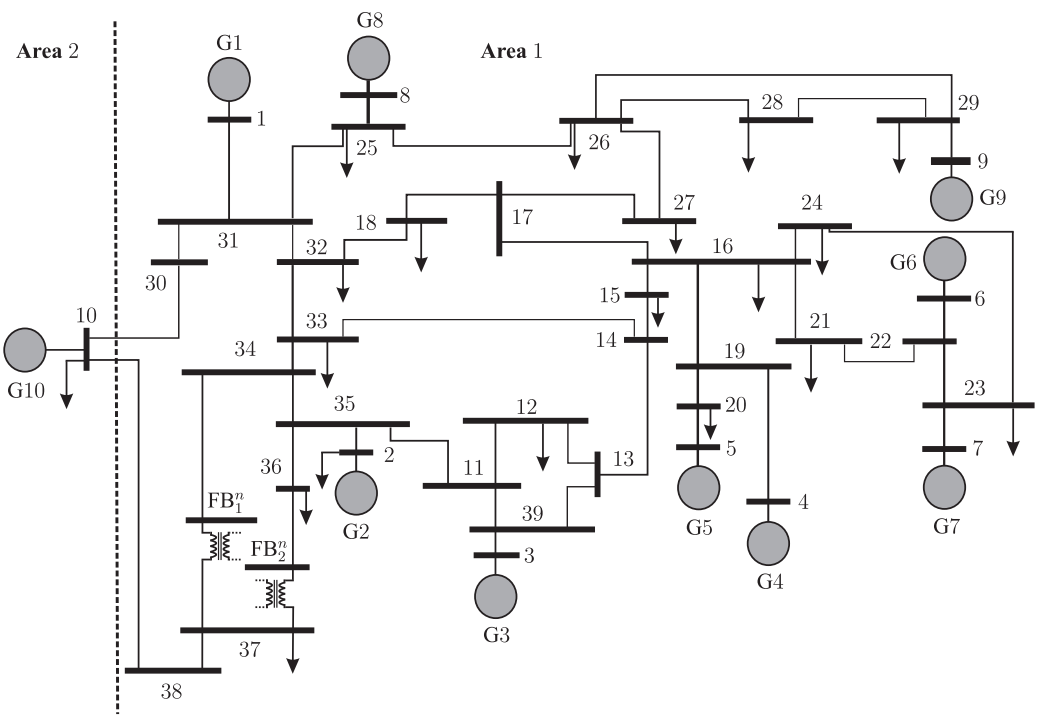

(c)

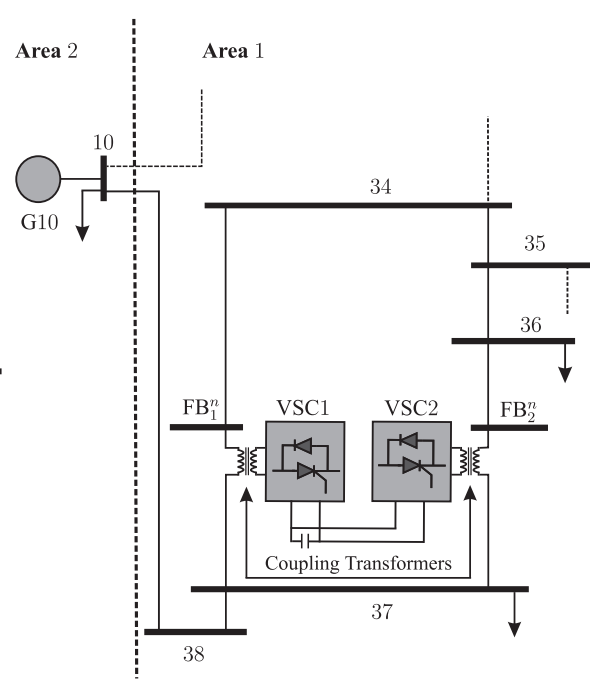

(d)

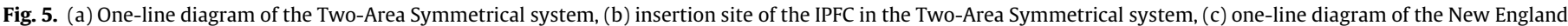
system, and (d) insertion site of the IPFC in New England system.

In Figs. 5(b) and (d), it can be seen that the IPFC device was installed between buses 7 and 8 in the Two-Area Symmetrical system and between buses 34, 36, and 37 in the New England system. These locations were determined by technical factors. In the case of the Two-Area Symmetrical system, there are three transmission lines between buses 7 and 8 . As the IPFC was installed in two lines to carry out the control of the flows, there was the possibility of having a slack line. Moreover, these are long lines, that are in parallel, with high inductive reactances, which favors the appearance of an inter-area oscillation mode. Finally, the voltage profile of the base case shown in Fig. 7(a) indicated that the voltage magnitude at bus 7 was lower than 0.95 p.u. (0.94 p.u.). It was expected that the IPFC device would improve the voltage profile at this bus, through the control of active and reactive power flows. In the New England system, the ideal place to install the IPFC device would be in the interconnection line between buses 10 and 38 .
As there is only a line between these buses, it was impractical to install the device on this site; therefore, it was installed between buses 34,36 , and 37 . The low voltage problems at buses 12,15 , 33, 36, and 37, lower than 0.95 p.u. (see Fig. 7(b)), also justified the installation of the IPFC at this location.

With the actuation of the IPFC device that controls active and reactive power flows on both the Two-Area Symmetrical system and the New England system, it can be verified that a new voltage profile was achieved for each one of these systems (Fig. 7(a) and (b)). In particular, the voltage magnitudes at bus 7 of the TwoArea Symmetrical system, and at buses 12, 15, 33, 36, and 37 of the New England system were improved, within the acceptable limits ( $\pm 5 \%$ of the nominal value).

For the results shown in Fig. 7(a) and (b), the flows on lines $7-\mathrm{FB}_{1}^{k}$ and $7-\mathrm{FB}_{2}^{k}$ of the Two-Area Symmetrical system were controlled (see Fig. 5(a) and (b)) by the IPFC device, with the following 


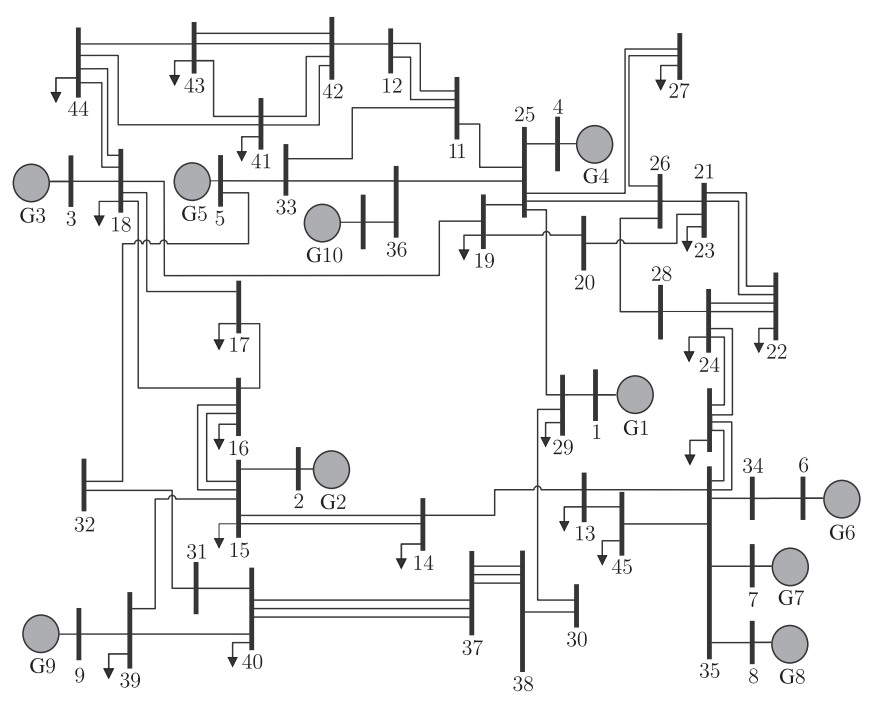

Fig. 6. One-line diagram of the Reduced Southern Brazilian system.

values (Case B): $\quad P_{j}^{r e f}=75.83 \mathrm{MW} \quad$ (Case A: $64.81 \mathrm{MW}$ ), $Q_{j}^{r e f}=-79.87 \mathrm{MVAr}$ (Case A: $-24.96 \mathrm{MW}$ ), and $P_{k}^{r e f}=75.83 \mathrm{MW}$ (Case A: $64.81 \mathrm{MW})$. For the New England system, the flows on lines $\mathrm{FB}_{1}^{n}-34$ and $\mathrm{FB}_{2}^{n}-36$ (see Fig. 5(c) and (d)) were controlled, with the following values (Case B): $P_{j}^{\text {ref }}=-410.51 \mathrm{MW}$ (Case A: $-310.98 \mathrm{MW}$ ), $Q_{j}^{\text {ref }}=-55.81 \mathrm{MVAr}$ (Case A: $-42.28 \mathrm{MVAr}$ ), and $P_{k}^{r e f}=-316.71 \mathrm{MW}$ (Case A: $-241.76 \mathrm{MW}$ ).

Figs. 8 and 9 show the active power flows in the Two-Area Symmetrical system and in the New England system (see Fig. 5) with and without the operation of the IPFC.

By analyzing the active power flows after the operation of the IPFC shown in Figs. 8 and 9, it is possible to verify that the device does not provide active power to the power system (active power invariance constraint of the IPFC), but it transfers, through the serial converters, active power for the lines on which the flows are controlled.

Regarding the Reduced Southern Brazilian system, there was no need to install an IPFC-POD device in this system. Two reasons justify this decision: first, as will be shown in Section 6.4, the system did not have any inter-area mode of oscillation and, thus, as will be proven, the PSS devices were enough to insert damping to the local mode electromechanical oscillations present in the electric power system; second, in Fig. 7(c), it can be seen that the voltage magnitudes at all buses were greater than 0.95 p.u. and, therefore, there was no need for an improvement of the voltage profile of this system.

\subsection{Performance of the VNS algorithm}

To evaluate the performance of the algorithms, the following was considered: (1) 100 tests limited to 2000 evaluations of the objective function; (2) the algorithms should be able to allocate

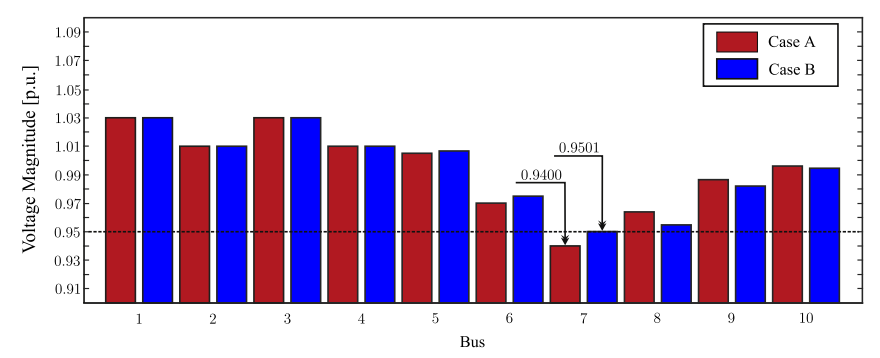

(a)

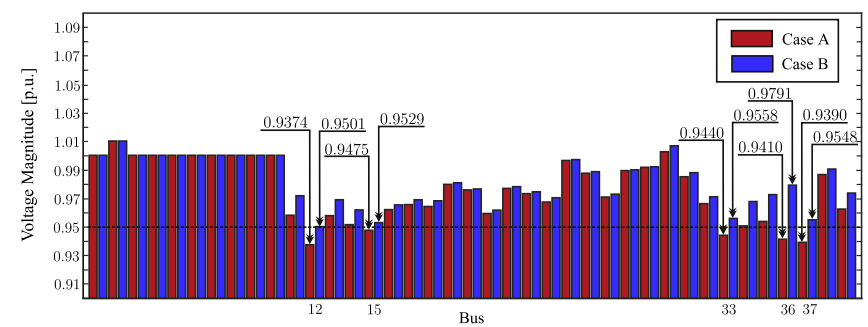

(b)

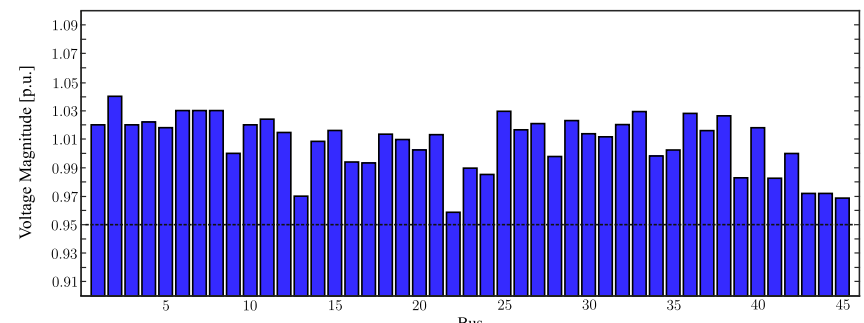

(c)

Fig. 7. Profiles of the voltage magnitudes of the (a) Two-Area Symmetrical system, (b) New England system, and (c) Reduced Southern Brazilian system.

all poles of interest with at least $10 \%$ of damping; (3) Repeat Case (2), considering at least $15 \%$ of damping for all modes of interest.

In the tests with the VNS and the multi-start algorithms, it was considered the evaluation function shown in (19). In all simulations (see Table 3), it was considered that the parameters of the controllers should satisfy the bounds shown in Table 1.

In Table 3, only the percentage of times that each algorithm has achieved convergence is shown. In addition, for the number of iterations (average, maximum, median, and standard deviation), only the tests in which the algorithms converged were considered.

For the Two-Area Symmetrical system, both algorithms had similar performance, converging for $100 \%$ of the tests. The difference between them, in this case, was related to the number of iterations necessary to achieve convergence. For both damping ranges ( $\xi_{i}=10 \%$ and $\xi_{i}=15 \%$ ), on average and when the maximum number of iterations are compared, the VNS algorithm performance is better than the multi-start. The medians and the standard deviations also indicate better convergence rates for the VNS algorithm.

For the New England system, the VNS algorithm performed better for both damping ranges on the average, converging for the

Table 2

Variables of the IPFC's control structure.

\begin{tabular}{|c|c|c|c|c|}
\hline IPFC & $V_{j}^{p}$ [p.u.] & $V_{k}^{p}$ [p.u.] & $V_{j}^{q}$ [p.u.] & $V_{k}^{q}$ [p.u.] \\
\hline \multicolumn{5}{|c|}{ Two-Area Symmetrical System } \\
\hline Case A & 0.0000 & 0.0000 & 0.0000 & 0.0000 \\
\hline Case B & 0.1187 & 0.0545 & -0.2406 & 0.0964 \\
\hline \multicolumn{5}{|c|}{ New England System } \\
\hline Case A & 0.0000 & 0.0000 & 0.0000 & 0.0000 \\
\hline Case B & -0.2938 & -0.3049 & -0.0485 & 0.0048 \\
\hline
\end{tabular}




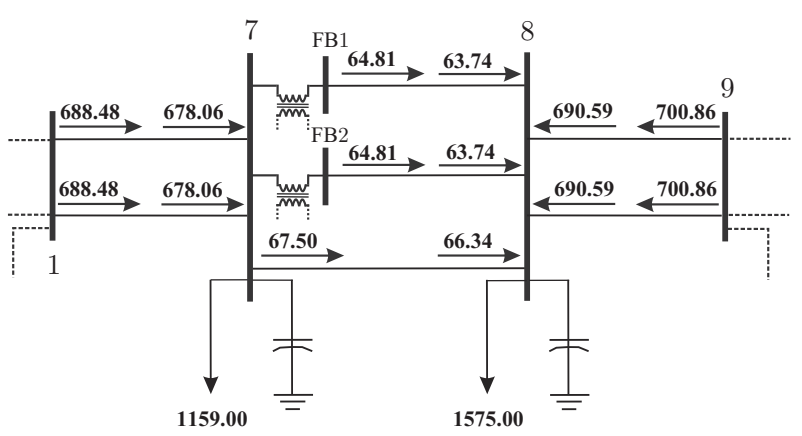

(a)

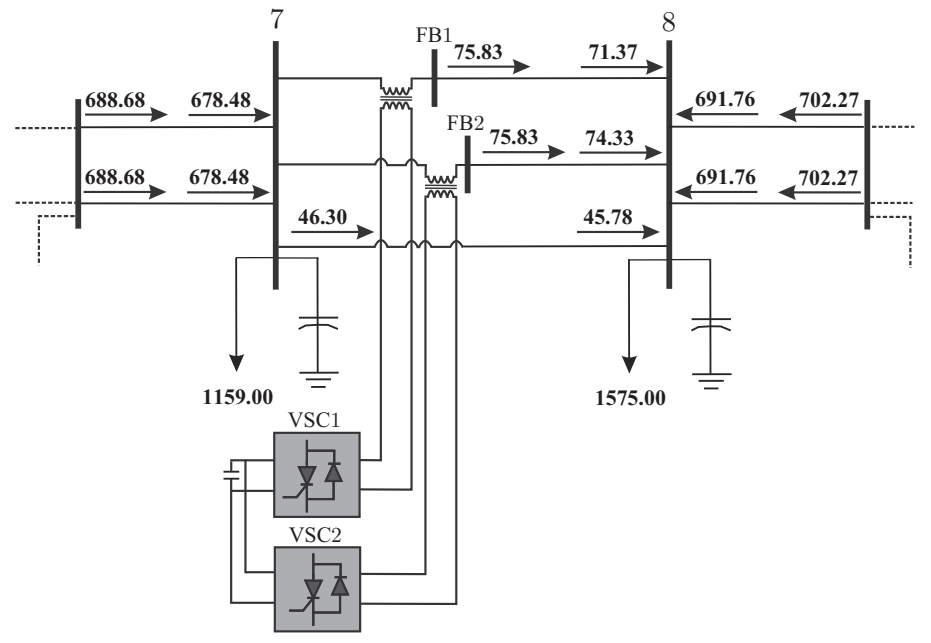

(b)

Fig. 8. Active power flow control performed by the IPFC in the Two-Area Symmetrical system. System (a) without the IPFC, and (b) with the IPFC.

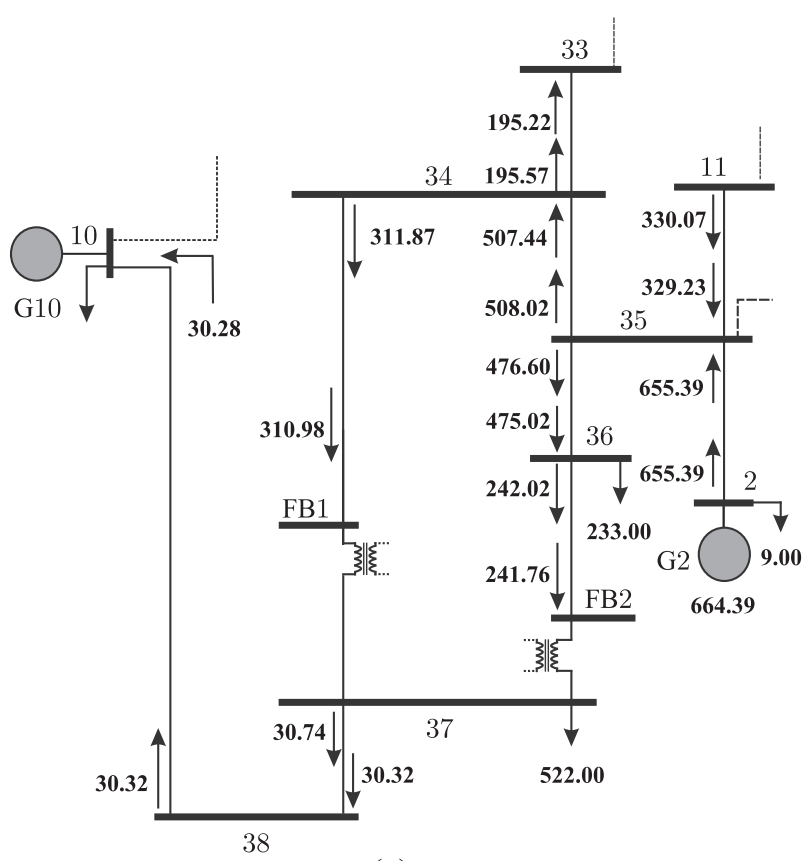

(a)

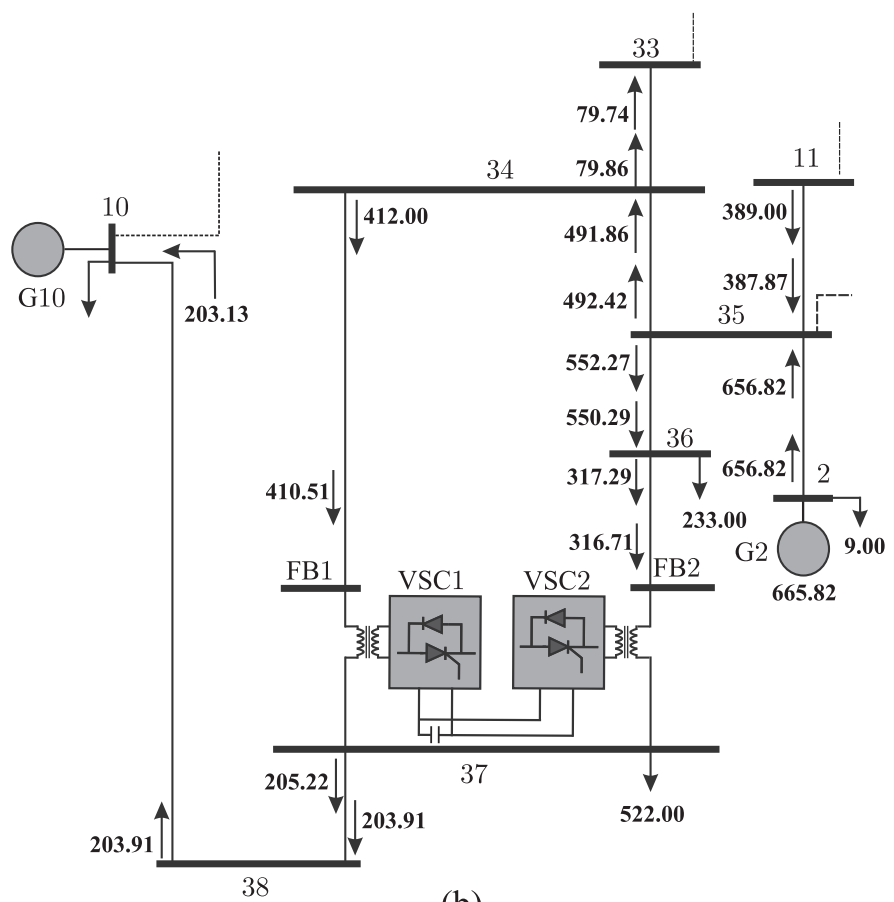

(b)

Fig. 9. Active power flow control performed by the IPFC in the New England system. System (a) without the IPFC, and (b) with the IPFC.

solution on $100 \%$ of the tests. For the second damping range, the multi-start algorithm converged for $96 \%$ of the tests.

Finally, for the Reduced Southern Brazilian system, for the first damping range $\left(\underline{\xi}_{i}=10 \%\right)$, it can be observed that, on average, the VNS algorithm converged in one third of the iterations of the multi-start method. Also, for the damping range $\underline{\xi}_{i}=15 \%$, the VNS converged for $79 \%$ of the tests, while the multi-start converged for $50 \%$ of the tests.

Therefore, the statistical metrics presented in Table 3 indicate superior performance of the VNS metaheuristic when it is compared to the multi-start algorithm for optimizing the considered problem.

\subsection{Dynamic analysis}

This subsection presents the results for the dynamic analysis performed for the three systems considered.
In this paper, the parameters of the PI controllers of the dynamic model of the IPFC (see Fig. 2) were fixed, and they can be seen in Table 4 .

\subsubsection{Two-Area Symmetrical system}

For this system, the tests are separated in Case A (without the actuation of the IPFC device to control the power flows, and not considering the POD and PSS controllers in the system) and Case $\mathrm{B}$ (with the actuation of the IPFC device). For Case B, three different scenarios were considered (besides the base case in which the PSS and POD controllers are not considered):

1. Scenario I: Considering only the installation of the IPFC-POD controller;

2. Scenario II: Considering only the installation of the two PSS controllers; 
Table 3

Comparison the algorithms' performances.

\begin{tabular}{|c|c|c|c|c|c|c|}
\hline \multirow[t]{2}{*}{ Algorithm } & \multirow[t]{2}{*}{$\underline{\xi}_{i}[\%]$} & \multirow[t]{2}{*}{ Converged tests [\%] } & \multicolumn{4}{|c|}{ Number of iterations } \\
\hline & & & Average & Maximum & Median & Standard deviation \\
\hline \multicolumn{7}{|c|}{ Two-Area Symmetrical System } \\
\hline \multirow[t]{2}{*}{ VNS } & 10 & 100 & 43 & 65 & 41 & 9 \\
\hline & 15 & 100 & 78 & 105 & 55 & 15 \\
\hline \multirow[t]{2}{*}{ Multi-Start } & 10 & 100 & 52 & 140 & 49 & 28 \\
\hline & 15 & 100 & 85 & 353 & 67 & 55 \\
\hline \multicolumn{7}{|c|}{ New England System } \\
\hline \multirow[t]{2}{*}{ VNS } & 10 & 100 & 164 & 270 & 165 & 27 \\
\hline & 15 & 100 & 449 & 1991 & 381 & 234 \\
\hline \multirow[t]{2}{*}{ Multi-Start } & 10 & 100 & 294 & 1141 & 219 & 209 \\
\hline & 15 & 96 & 607 & 1659 & 427 & 365 \\
\hline \multicolumn{7}{|c|}{ Reduced Southern Brazilian System } \\
\hline \multirow[t]{2}{*}{ VNS } & 10 & 100 & 175 & 1465 & 89 & 95 \\
\hline & 15 & 79 & 677 & 1902 & 382 & 307 \\
\hline \multirow[t]{2}{*}{ Multi-Start } & 10 & 98 & 577 & 1905 & 497 & 441 \\
\hline & 15 & 50 & 867 & 1978 & 767 & 532 \\
\hline
\end{tabular}

Table 4

Parameters of the PI controllers of the dynamic model of the IPFC.

\begin{tabular}{|c|c|c|c|c|c|c|}
\hline System & $T_{1}^{p i}[\mathrm{~s}]$ & $T_{2}^{p i}[\mathrm{~s}]$ & $T_{3}^{p i}[\mathrm{~s}]$ & $K_{1}^{p i}$ [p.u.] & $K_{2}^{p i}$ [p.u.] & $K_{3}^{p i}$ [p.u.] \\
\hline Two-Area Symmetrical System & 0.0872 & 0.0450 & 0.1000 & 3.3492 & 2.6010 & 1.2761 \\
\hline New England System & 0.0074 & 0.0052 & 0.0056 & 0.8357 & 0.7555 & 0.9880 \\
\hline
\end{tabular}

Table 5

Parameters of the PSS and IPFC-POD controllers adjusted by the VNS algorithm.

\begin{tabular}{|c|c|c|c|c|c|}
\hline \multirow[t]{2}{*}{ Scenario } & & \multirow[t]{2}{*}{ Parameters } & \multicolumn{3}{|c|}{ Device } \\
\hline & & & PSS G1 & PSS G2 & IPFC-POD \\
\hline \multirow[t]{3}{*}{ I } & & $T_{1}^{p o d}[\mathrm{~s}]$ & - & - & 1.0000 \\
\hline & & $T_{2}^{\text {pod }}[\mathrm{s}]$ & - & - & 0.4300 \\
\hline & & $K^{\text {pod }}$ [p.u.] & - & - & 0.8100 \\
\hline \multirow[t]{3}{*}{ II } & & $T_{1}^{p s s}[\mathrm{~s}]$ & 1.0000 & 0.5250 & - \\
\hline & & $T_{2}^{p s s}[\mathrm{~s}]$ & 0.7150 & 0.2400 & - \\
\hline & & $K^{p s s}$ [p.u.] & 2.2000 & 1.4500 & - \\
\hline \multirow[t]{6}{*}{ III } & $\underline{\xi}_{i}=10 \%$ & $T_{1}^{p s s} / T_{1}^{p o d}[\mathrm{~s}]$ & 0.7750 & 0.7600 & 0.9600 \\
\hline & & $T_{2}^{p s s} / T_{2}^{p o d}[\mathrm{~s}]$ & 0.6400 & 0.5600 & 0.6400 \\
\hline & & $K^{p s s} / K^{p o d}[$ p.u.] & 3.4000 & 3.8000 & 1.2000 \\
\hline & $\underline{\xi}_{i}=15 \%$ & $T_{1}^{p s s} / T_{1}^{p o d}[s]$ & 1.0000 & 1.0000 & 0.9000 \\
\hline & & $T_{2}^{p s s} / T_{2}^{p o d}[\mathrm{~s}]$ & 0.7000 & 0.6200 & 0.5200 \\
\hline & & $K^{\text {pss }} / K^{\text {pod }}[$ [p.u.] & 4.5000 & 4.6000 & 1.3000 \\
\hline
\end{tabular}

3. Scenario III: Considering the installation of the two PSS and the IPFC-POD controller.

The purpose of these tests is to show that the PSS controllers have great impact on local oscillation modes, while the IPFC-POD has great influence in the inter-area mode. The minimum damping restriction of $10 \%\left(\xi_{i}=10 \%\right)$ was used on the modes of interest for Scenarios I and II, and $10 \%$ and $15 \%\left(\underline{\xi_{i}}=15 \%\right)$ was imposed on the modes of interest for the tests in Scenario III.

The parameters of the controllers, obtained by the VNS algorithm, for the three scenarios are presented in Table 5. From the results obtained with the parameters for Scenarios I, II, and III,

Table 6

Damping ratios and natural undamped frequencies of the Two-Area Symmetrical system.

\begin{tabular}{|c|c|c|c|c|c|c|c|c|c|c|c|c|}
\hline \multirow[t]{3}{*}{ Mode } & \multicolumn{2}{|c|}{ Case A } & \multicolumn{10}{|c|}{ Case B } \\
\hline & \multirow[b]{2}{*}{$\xi_{i}$ [p.u.] } & \multirow[b]{2}{*}{$\omega_{n_{i}}[\mathrm{~Hz}]$} & \multicolumn{2}{|c|}{ Base Case } & \multicolumn{2}{|c|}{ Scenario I } & \multicolumn{2}{|c|}{ Scenario II } & \multicolumn{4}{|c|}{ Scenario III } \\
\hline & & & $\xi_{i}$ [p.u.] & $\omega_{n_{i}}[\mathrm{~Hz}]$ & $\xi_{i}$ [p.u.] & $\omega_{n_{i}}[\mathrm{~Hz}]$ & $\xi_{i}$ [p.u.] & $\omega_{n_{i}}[\mathrm{~Hz}]$ & $\xi_{i}[$ p.u.] & $\omega_{n_{i}}[\mathrm{~Hz}]$ & $\xi_{i}$ [p.u.] & $\omega_{n_{i}}[\mathrm{~Hz}]$ \\
\hline$\lambda_{1}$ & 0.0544 & 1.0035 & 0.0578 & 1.0041 & 0.0548 & 1.0061 & 0.1065 & 1.0329 & 0.1091 & 1.0442 & 0.1510 & 1.0736 \\
\hline$\lambda_{2}$ & 0.0472 & 0.9409 & 0.0443 & 0.9419 & 0.0441 & 0.9427 & 0.1036 & 0.9435 & 0.1069 & 0.9813 & 0.1543 & 1.0102 \\
\hline$\lambda_{3}$ & -0.0112 & 0.7063 & -0.0118 & 0.7112 & 0.1004 & 0.5908 & 0.0019 & 0.7108 & 0.1018 & 0.6340 & 0.1506 & 0.6035 \\
\hline
\end{tabular}


new dominant eigenvalues $\left(\lambda_{i}=\sigma_{i} \pm \omega_{i}\right)$, damping coefficients $\left(\xi_{i}=-\frac{\sigma_{i}}{\left|\lambda_{i}\right|}\right)$, and natural undamped frequencies $\left(\omega_{n_{i}}=\frac{\left|\lambda_{i}\right|}{2 \pi}\right)$ were found for the Two-Area Symmetrical system. These results are presented in Table 6 along with the results for Case A and for the base case of Case B.

By analyzing the natural undamped frequencies of the eigenvalues shown in Table 6 for Case A, the existence of two local modes $\left(\lambda_{1}\right.$ and $\left.\lambda_{2}\right)$ and an inter-area mode $\left(\lambda_{3}\right)$ with positive real part can be verified, which characterizes the system as unstable.

Moreover, comparing the damping coefficients associated with the three oscillation modes before (Case A) and after (base case of Case B) the actuation of the IPFC device, a slight improvement of the damping associated with the local mode $\lambda_{1}$ can be verified. However, the device actuation negatively influenced the damping of the other two modes $\left(\lambda_{2}\right.$ and $\left.\lambda_{3}\right)$.

For Case B, Scenario I, in the considered operating point, the test system was stable and the inter-area mode $\left(\lambda_{3}\right)$ had more than $10 \%$ of damping. In this case, the damping of the local modes $\lambda_{1}$ and $\lambda_{2}$ remained almost unchanged, indicating that the IPFC-POD controller operated specifically in the inter-area oscillation mode. When Scenario II was considered, it was noticed that the joint action of the PSS controllers, on generators G2 and G3, inserted damping (greater than $10 \%$ ) to local modes. Although the system becomes stable, because the inter-area mode $\left(\lambda_{3}\right)$ had negative real part, it was verified that the PSS controllers had a small influence in this oscillation mode. Under these conditions, small topological or parametric variations can lead to the system's instability. Finally, there was Scenario III, in which the joint action of the IPFC-POD and PSS controllers on generators G2 and G3 was effective. The system became stable, and all modes of interest of the system presented damping greater than $10 \%$ and $15 \%$ in the two sub-cases analyzed for Scenario III, i.e., the system presented a high margin of stability for the operating point considered.

\subsubsection{Reduced Southern Brazilian system}

The results for the base case presented in Table 7 indicates the presence of nine oscillation modes in the Reduced Southern Brazilian system. The presence of five modes with positive real part $\left(\lambda_{1}, \lambda_{3}, \lambda_{4}, \lambda_{7}\right.$, and $\left.\lambda_{8}\right)$ classified the system as unstable for the point of operation considered. Moreover, after the analysis of the natural undamped frequency of each mode of interest, each oscillation mode of the system can be classified as local.

Table 7

Damping ratios and natural undamped frequencies of the Reduced Southern Brazilian system.

\begin{tabular}{|c|c|c|c|c|c|c|}
\hline \multirow[t]{2}{*}{ Mode } & \multicolumn{2}{|c|}{ Base Case } & \multicolumn{2}{|c|}{$\underline{\xi}_{i}=10 \%$} & \multicolumn{2}{|c|}{$\underline{\xi_{i}}=15 \%$} \\
\hline & $\xi_{i}$ [p.u.] & $\omega_{n_{i}}[\mathrm{~Hz}]$ & $\xi_{i}$ [p.u.] & $\omega_{n_{i}}[\mathrm{~Hz}]$ & $\xi_{i}$ [p.u.] & $\omega_{n_{i}}[\mathrm{~Hz}]$ \\
\hline$\lambda_{1}$ & -0.0144 & 1.1676 & 0.1025 & 1.2651 & 0.1819 & 0.9184 \\
\hline$\lambda_{2}$ & 0.0025 & 1.2531 & 0.1047 & 1.0072 & 0.2833 & 1.1419 \\
\hline$\lambda_{3}$ & -0.0140 & 1.0276 & 0.1098 & 1.3174 & 0.1500 & 1.2618 \\
\hline$\lambda_{4}$ & -0.0185 & 1.2961 & 0.1027 & 1.1350 & 0.1500 & 1.2221 \\
\hline$\lambda_{5}$ & 0.0295 & 1.5621 & 0.1456 & 1.4591 & 0.2914 & 1.6518 \\
\hline$\lambda_{6}$ & 0.0141 & 1.5511 & 0.1081 & 1.6180 & 0.1859 & 0.9653 \\
\hline$\lambda_{7}$ & -0.0027 & 1.1465 & 0.1015 & 1.0925 & 0.2345 & 0.9535 \\
\hline$\lambda_{8}$ & -0.0120 & 1.0936 & 0.1006 & 1.0546 & 0.1837 & 1.0478 \\
\hline$\lambda_{9}$ & 0.0060 & 1.3926 & 0.1163 & 1.4578 & 0.2187 & 1.7364 \\
\hline
\end{tabular}

Table 8

Gains and time constants of the PSS controllers adjusted by the VNS algorithm: Reduced Southern Brazilian system.

\begin{tabular}{|c|c|c|c|c|c|c|}
\hline \multirow[t]{2}{*}{ Device } & \multicolumn{3}{|c|}{$\underline{\xi}_{i}=10 \%$} & \multicolumn{3}{|c|}{$\underline{\xi}_{i}=15 \%$} \\
\hline & $T_{1}^{p s s}[\mathrm{~s}]$ & $T_{2}^{p s s}[\mathrm{~s}]$ & $K^{p s s}[$ p.u.] & $T_{1}^{p s s}[\mathrm{~s}]$ & $T_{2}^{p s s}[\mathrm{~s}]$ & $K^{p s s}$ [p.u.] \\
\hline PSS G1 & 0.1500 & 0.0400 & 5.8000 & 0.3875 & 0.1156 & 3.5657 \\
\hline PSS G2 & 0.1100 & 0.1000 & 3.9000 & 0.1213 & 0.0611 & 11.0406 \\
\hline PSS G3 & 0.1240 & 0.0700 & 7.4400 & 0.4780 & 0.1878 & 3.1835 \\
\hline PSS G4 & 0.1100 & 0.0590 & 9.9200 & 0.2469 & 0.1615 & 10.9989 \\
\hline PSS G6 & 0.1000 & 0.0820 & 4.0150 & 0.1399 & 0.1243 & 8.0107 \\
\hline PSS G7 & 0.0680 & 0.0900 & 2.3000 & 0.2878 & 0.0760 & 6.3876 \\
\hline PSS G8 & 0.1550 & 0.0280 & 4.1400 & 0.1553 & 0.0539 & 5.1054 \\
\hline PSS G9 & 0.1100 & 0.0420 & 6.4000 & 0.2562 & 0.1590 & 7.9824 \\
\hline PSS G10 & 0.0850 & 0.0970 & 5.5000 & 0.2031 & 0.2179 & 11.8336 \\
\hline
\end{tabular}

Table 9

Damping ratios and natural undamped frequencies of the New England system.

\begin{tabular}{|c|c|c|c|c|c|c|c|c|}
\hline \multirow[t]{3}{*}{ Mode } & \multicolumn{2}{|c|}{ Case A } & \multicolumn{6}{|c|}{ Case B } \\
\hline & \multirow[b]{2}{*}{$\xi_{i}$ [p.u.] } & \multirow[b]{2}{*}{$\omega_{n_{i}}[\mathrm{~Hz}]$} & \multicolumn{2}{|c|}{ Base Case } & \multicolumn{2}{|c|}{$\underline{\xi_{i}}=10 \%$} & \multicolumn{2}{|c|}{$\underline{\xi}_{i}=15 \%$} \\
\hline & & & $\xi_{i}$ [p.u.] & $\omega_{n_{i}}[\mathrm{~Hz}]$ & $\xi_{i}$ [p.u.] & $\omega_{n_{i}}[\mathrm{~Hz}]$ & $\xi_{i}$ [p.u.] & $\omega_{n_{i}}[\mathrm{~Hz}]$ \\
\hline$\lambda_{1}$ & -0.0100 & 1.0918 & -0.0070 & 1.0922 & 0.1134 & 1.0087 & 0.1528 & 1.1759 \\
\hline$\lambda_{2}$ & 0.0282 & 1.1609 & 0.0301 & 1.1637 & 0.1258 & 1.1212 & 0.1980 & 1.0427 \\
\hline$\lambda_{3}$ & 0.0235 & 1.3159 & 0.0243 & 1.3169 & 0.1421 & 1.0649 & 0.1540 & 1.0346 \\
\hline$\lambda_{4}$ & -0.0251 & 0.9490 & -0.0253 & 0.9489 & 0.1291 & 1.1525 & 0.1542 & 1.2779 \\
\hline$\lambda_{5}$ & 0.0184 & 1.0330 & 0.0184 & 1.0369 & 0.1006 & 1.1379 & 0.1659 & 1.2166 \\
\hline$\lambda_{6}$ & 0.0332 & 1.2901 & 0.0207 & 1.2914 & 0.1094 & 1.2544 & 0.1932 & 1.0449 \\
\hline$\lambda_{7}$ & 0.0292 & 1.3244 & 0.0289 & 1.3251 & 0.1004 & 0.9058 & 0.1681 & 0.9714 \\
\hline$\lambda_{8}$ & -0.0226 & 1.0151 & -0.0284 & 1.0170 & 0.1165 & 0.8118 & 0.1500 & 0.7600 \\
\hline$\lambda_{9}$ & -0.0085 & 0.6152 & -0.0075 & 0.6150 & 0.1110 & 0.6720 & 0.1683 & 0.6428 \\
\hline
\end{tabular}


After processing the VNS algorithm, the parameters of the supplementary damping controllers were obtained (Table 8).

In Table 7, the adjustments made for the parameters of the PSS controllers by the VNS algorithm satisfied the two minimum damping restrictions for the local oscillation modes: $\xi_{i}=10 \%$ and $\underline{\xi}_{i}=15 \%$. With the parameters shown in Table 8, new dominant eigenvalues, damping coefficients, and natural undamped frequencies were calculated for the system. Table 7 shows the two new operating states for the Reduced Southern Brazilian system, both with at least $10 \%$ and $15 \%$ of damping for the system's modes of interest (local modes).

The new operating states obtained for the Reduced Southern Brazilian system $\left(\xi_{i}=10 \%\right.$ and $\left.\xi_{i}=15 \%\right)$, after the adjustments of the parameters of the PSS controllers, carried out by the VNS algorithm, indicated that the system was stable and operating with high levels of damping. The results obtained from the simulations performed with this test system allow us to confirm the conclusions of Section 6.4.1, i.e., PSS controllers are efficient to insert damping to local oscillation modes; therefore, there was no need to install another supplementary damping controller in this system.

\subsubsection{New England system}

The tests for the New England system were separated in Case A (without the actuation of the IPFC device to control the power flows, and not considering the POD and PSS controllers in the system) and Case B (with the actuation of the IPFC device). The base case for Case B does not consider the PSS and POD controllers in the system. The results for this system are presented in Table 9.

Based on the analysis of Table 9, it can be concluded that the IPFC alone did not have good performance in damping the lowfrequency oscillations present in the New England system (base case of Case B). Improvements are noted in the damping of the local modes $\lambda_{4}, \lambda_{6}, \lambda_{7}$, and $\lambda_{8}$. The negative influence in the damping can be verified on local modes $\lambda_{1}, \lambda_{2}$, and $\lambda_{3}$, and on the inter-area mode $\lambda_{9}$. Local mode $\lambda_{5}$ maintained the same damping before and after the inclusion and actuation of the IPFC device for the control of active and reactive power flows in the New England system.

Table 10

Gains and time constants of the PSS and IPFC-POD controllers adjusted by the VNS algorithm: New England system.

\begin{tabular}{|c|c|c|c|c|c|c|}
\hline \multirow[t]{2}{*}{ Device } & \multicolumn{3}{|c|}{$\underline{\xi_{i}}=10 \%$} & \multicolumn{3}{|c|}{$\underline{\xi}_{i}=15 \%$} \\
\hline & $T_{1}^{p s s} / T_{1}^{p o d}[\mathrm{~s}]$ & $T_{2}^{p s s} / T_{2}^{p o d}[s]$ & $K^{p s s} / K^{\text {pod }}[$ p.u.] & $T_{1}^{p s s} / T_{1}^{p o d}[\mathrm{~s}]$ & $T_{2}^{p s s} / T_{2}^{p o d}[s]$ & $K^{p s s} / K^{p o d}[$ p.u.] \\
\hline PSS G1 & 1.4058 & 0.0289 & 6.6325 & 1.3250 & 0.0880 & 9.6218 \\
\hline PSS G2 & 0.5917 & 0.0387 & 5.0707 & 0.6200 & 0.0378 & 9.1768 \\
\hline PSS G3 & 0.6153 & 0.0474 & 6.0067 & 0.5291 & 0.0230 & 9.6135 \\
\hline PSS G4 & 0.5798 & 0.0165 & 6.2044 & 0.4907 & 0.0600 & 7.3980 \\
\hline PSS G6 & 0.4691 & 0.0571 & 6.2734 & 0.4839 & 0.0629 & 7.5139 \\
\hline PSS G7 & 0.3453 & 0.0391 & 4.8987 & 0.3971 & 0.0736 & 2.4905 \\
\hline PSS G8 & 0.5374 & 0.0414 & 4.6806 & 0.6408 & 0.0371 & 9.5487 \\
\hline PSS G9 & 0.3946 & 0.0583 & 1.8491 & 0.2792 & 0.0829 & 7.8125 \\
\hline IPFC-POD & 0.1138 & 0.1788 & 0.3088 & 0.1142 & 0.3848 & 0.4832 \\
\hline
\end{tabular}
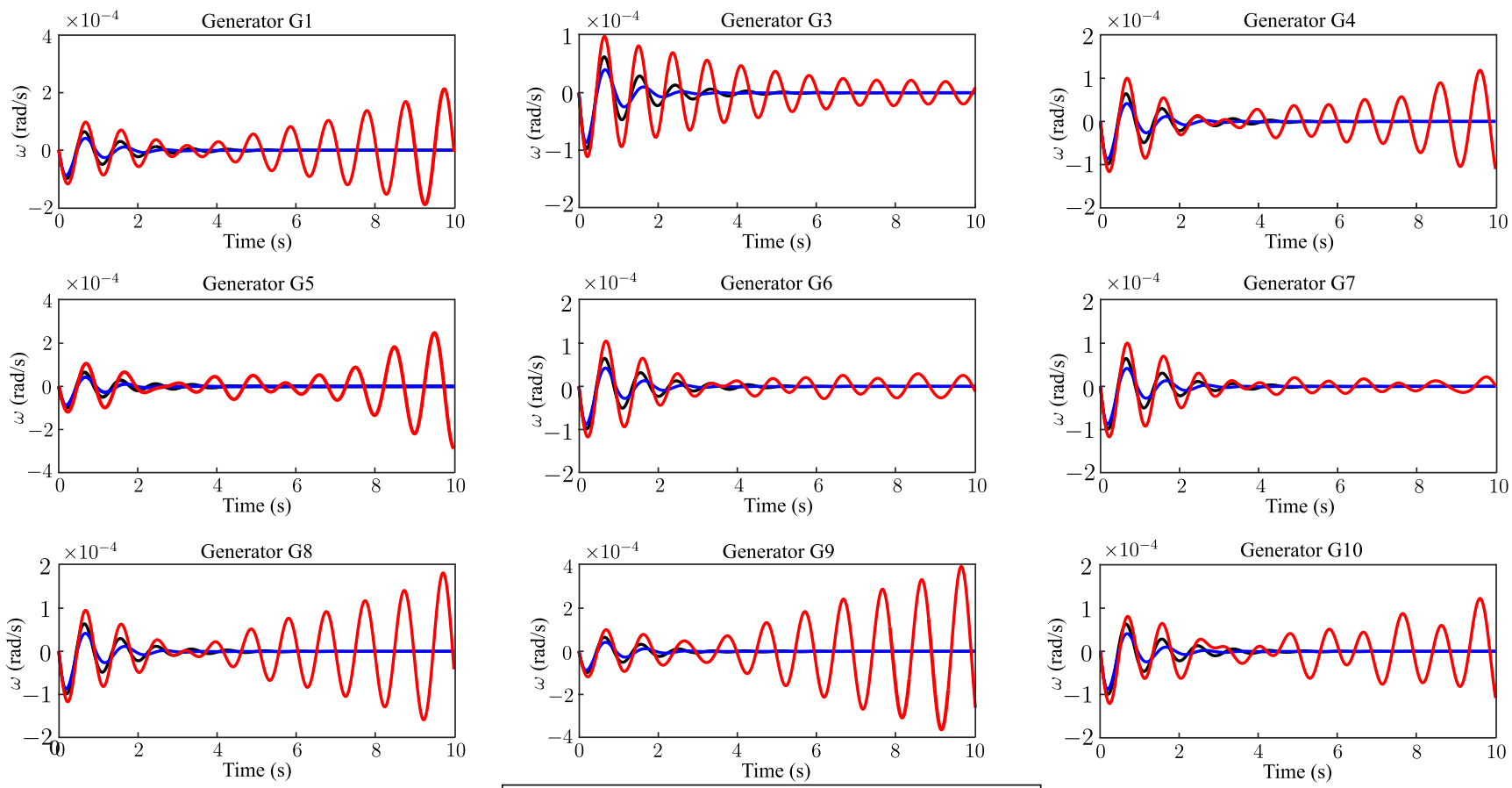

Base Case $\longrightarrow \underline{\xi}_{i}=15 \%-\underline{\xi}_{i}=10 \%$

Fig. 10. Variations of the angular velocity of generators G1, G3, G5, G6, G7, G8, G9, and G10 in the New England system. 
The New England system was characterized by having eight local oscillation modes and one inter-area mode. Thus, according to Sections 6.4.1 and 6.4.2, eight PSS and an IPFC-POD controllers were added in this system to insert damping to local and inter-area modes, respectively.

In Table 10, the results obtained by the VNS algorithm for the parameters of the PSS and IPFC-POD controllers to a valid state are shown, i.e., they satisfy (19) and the bounds shown in Table 1, which made it possible to modify the damping levels for the base case of Case B shown in Table 9 to values greater than or equal to $10 \%\left(\underline{\xi}_{i}=10 \%\right)$ and $15 \%\left(\underline{\xi}_{i}=15 \%\right)$ for all oscillation modes, i.e., local and inter-area modes.

By setting the New England system with the parameters of the PSS and IPFC-POD controllers according to Table 10, new dominant eigenvalues, damping coefficients, and natural undamped frequencies were calculated, obtained through simulations and shown in Table 9 with two new operating states, both with at least $10 \%$ and $15 \%$ of damping for the modes of interest.

The new operating states obtained for the New England system presented in Table 9 ( $\underline{\xi}_{i}=10 \%$ and $\left.\underline{\xi}_{i}=15 \%\right)$ show a high damping margin for this test system and confirm that the joint action of the PSS and the IPFC-POD is able to insert damping to local and interarea modes of oscillation when their control parameters are designed correctly.

Fig. 10 shows the variations of the angular velocities of generators G1, G3, G4, G5, G6, G7, G8, G9, and G10 of the New England system in relation to the variation of the angular velocity of gener-

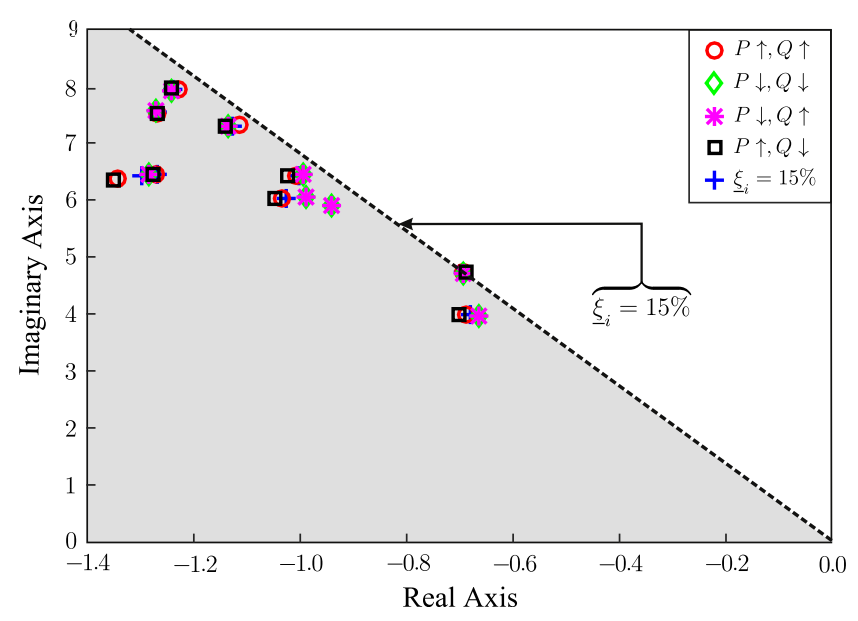

Fig. 11. Positioning of the eigenvalues of interest on the complex plane for the New England system. ator G2, the reference in the system, in which a step of 0.05 p.u. was applied to the mechanical power. In the cases presented in Fig. 10, the three scenarios already analyzed for Case B were considered (see Table 9): Base Case, $\underline{\xi}_{i}=10 \%$, and $\underline{\xi}_{i}=15 \%$.

The electric power systems are subject to loading variations and consequent generation adjustments. The perturbations on the mechanical powers of the generators shown in Fig. 10 can be compared to these small generation adjustments. The curves in red represent the Base Case, while the black $\left(\underline{\xi}_{i}=10 \%\right)$ and blue $\left(\xi_{i}=15 \%\right)$ curves represent the cases in which the system operates with high stability margin, equipped with eight PSS controllers and one IPFC-POD set, with parameters designed using the VNS algorithm. By analyzing Fig. 10, it can be verified that the curves representing the Base Case, after the applied perturbation, are characterized by increasing or low damped amplitudes, typical of an unstable system (see Table 9). In the other two cases, even after the perturbation, the analysis of the obtained curves shows that the system is well damped, evidencing its high margin of stability to small-signal perturbations.

In order to verify the robustness of the parameters provided by the VNS algorithm, different loading scenarios are considered. The objective is to perform an analysis of the displacements of the oscillation modes of interest of the New England system (see Fig. 11). The loading scenarios are the following: $P \uparrow, Q \downarrow$ : an increase of $5 \%$ on the active loads and a decrease of $5 \%$ on the reactive loads; $P \downarrow, Q \uparrow:$ a decrease of $5 \%$ on the active loads and an increase $5 \%$ on the reactive loads; $P \downarrow, Q \downarrow$ : a decrease of $5 \%$ on both active and reactive loads; $P \uparrow, Q \uparrow:$ an increase of $5 \%$ on both active and reactive loads.

By analyzing Fig. 11, it can be verified that the damping levels in the New England system remained almost unchanged, even after the variations of the loads. In other words, the New England system was still able to operate with high damping levels for different load scenarios, evidencing the robustness of the parameters provided by the VNS algorithm.

\section{Conclusion}

In this paper, a variable neighborhood search algorithm for the coordinated design of the parameters of supplementary damping controllers (power system stabilizers and interline power flow controller-power oscillation damping controller) was presented. The objective was to insert damping to small-signal local and inter-area oscillation modes present in the power system. The effectiveness of the technique was evaluated using the Two-Area Symmetrical, the New England, and the Reduced Southern Brazilian systems.

Table 11

Synchronous generators and excitation system data for the Reduced Southern Brazilian system.

\begin{tabular}{|c|c|c|c|c|c|c|c|c|}
\hline \multirow[t]{2}{*}{ Generator $(k)$} & \multicolumn{6}{|c|}{ Synchronous generators } & \multicolumn{2}{|c|}{ Excitation system } \\
\hline & $x_{d_{k}}^{\prime}$ [p.u.] & $x_{d_{k}}$ [p.u.] & $x_{q_{k}}$ [p.u.] & $H_{k}[\mathrm{~s}]$ & $D_{k}$ [p.u.] & $T_{d_{0 k}}^{\prime}[\mathrm{s}]$ & $K_{k}^{r}$ [p.u.] & $T_{k}^{r}[\mathrm{~s}]$ \\
\hline 1 & 0.0457 & 0.1460 & 0.1050 & 37.32 & 0.10 & 6.90 & 200.00 & 0.001 \\
\hline 2 & 0.0985 & 0.4015 & 0.2979 & 11.30 & 0.00 & 4.90 & 200.00 & 0.001 \\
\hline 3 & 0.0300 & 0.0973 & 0.0700 & 39.46 & 0.00 & 8.27 & 200.00 & 0.001 \\
\hline 4 & 0.0180 & 0.0561 & 0.0400 & 74.40 & 0.00 & 7.92 & 200.00 & 0.001 \\
\hline 5 & 0.0215 & 0.0679 & 0.0500 & 57.28 & 0.00 & 10.00 & 200.00 & 0.001 \\
\hline 6 & 0.1350 & 1.0150 & 0.7700 & 3.56 & 0.00 & 5.00 & 200.00 & 0.001 \\
\hline 7 & 0.1535 & 0.9130 & 0.5304 & 5.06 & 0.00 & 4.89 & 200.00 & 0.001 \\
\hline 8 & 0.1007 & 0.4000 & 0.2987 & 9.68 & 0.00 & 6.66 & 200.00 & 0.001 \\
\hline 9 & 0.0550 & 0.2211 & 0.1235 & 20.34 & 0.00 & 9.55 & 200.00 & 0.001 \\
\hline 10 & 0.0184 & 0.0730 & 0.0525 & 51.63 & 0.00 & 7.59 & 200.00 & 0.001 \\
\hline
\end{tabular}


Table 12

Line and bus data for the Reduced Southern Brazilian system.

\begin{tabular}{|c|c|c|c|c|c|c|c|c|}
\hline Initial bus ( $i$ ) & End bus $(j)$ & $r_{i j}$ [p.u.] & $x_{i j}[$ p.u.] & $b_{i j}^{s h}$ [p.u.] & $P_{j}^{g}[\mathrm{MW}]$ & $Q_{j}^{g}[\mathrm{MVAr}]$ & $P_{j}^{l}[\mathrm{MW}]$ & $Q_{j}^{l}[\mathrm{MVAr}]$ \\
\hline 29 & 1 & 0.0000 & 0.0135 & 0.0000 & 650.00 & 4.92 & 0.00 & 0.00 \\
\hline 15 & 2 & 0.0000 & 0.0460 & 0.0000 & 215.00 & 64.24 & 0.00 & 0.00 \\
\hline 18 & 3 & 0.0000 & 0.0114 & 0.0000 & 895.00 & 102.90 & 0.00 & 0.00 \\
\hline 25 & 4 & 0.0000 & 0.0067 & 0.0000 & 1531.60 & -39.49 & 0.00 & 0.00 \\
\hline 33 & 5 & 0.0000 & 0.0114 & 0.0000 & 1325.00 & -4.78 & 0.00 & 0.00 \\
\hline 34 & 6 & 0.0000 & 0.0871 & 0.0000 & 90.00 & 41.09 & 0.00 & 0.00 \\
\hline 35 & 7 & 0.0000 & 0.0701 & 0.0000 & 120.00 & 45.51 & 0.00 & 0.00 \\
\hline 35 & 8 & 0.0000 & 0.0450 & 0.0000 & 241.00 & 75.96 & 0.00 & 0.00 \\
\hline 39 & 9 & 0.0000 & 0.0236 & 0.0000 & 460.00 & 98.12 & 0.00 & 0.00 \\
\hline 36 & 10 & 0.0000 & 0.0068 & 0.0000 & 1100.00 & -80.83 & 0.00 & 0.00 \\
\hline 12 & 11 & 0.0007 & 0.0145 & 1.5672 & 0.00 & 0.00 & 0.00 & 0.00 \\
\hline 11 & 12 & 0.0007 & 0.0145 & 1.5672 & 0.00 & 0.00 & 0.00 & 0.00 \\
\hline 14 & 13 & 0.0386 & 0.1985 & 0.3425 & 0.00 & 0.00 & 177.00 & 68.00 \\
\hline 15 & 14 & 0.0463 & 0.3378 & 0.3917 & 0.00 & 0.00 & 191.00 & 42.00 \\
\hline 14 & 15 & 0.0463 & 0.3378 & 0.3917 & 0.00 & 0.00 & 171.00 & 18.50 \\
\hline 15 & 16 & 0.0163 & 0.0835 & 0.1411 & 0.00 & 0.00 & 126.00 & 47.00 \\
\hline 16 & 17 & 0.0163 & 0.0835 & 0.1445 & 0.00 & 0.00 & 46.00 & 14.70 \\
\hline 16 & 18 & 0.0316 & 0.1621 & 0.2746 & 0.00 & 0.00 & 281.00 & 56.50 \\
\hline 18 & 19 & 0.0306 & 0.1523 & 0.2620 & 0.00 & 0.00 & 279.00 & 60.70 \\
\hline 19 & 20 & 0.0245 & 0.1256 & 0.1981 & 0.00 & 0.00 & 130.00 & 29.40 \\
\hline 20 & 21 & 0.0088 & 0.0415 & 0.5009 & 0.00 & 0.00 & 427.00 & -25.00 \\
\hline 23 & 22 & 0.0154 & 0.0776 & 0.1386 & 0.00 & 0.00 & 310.00 & 141.00 \\
\hline 22 & 23 & 0.0154 & 0.0776 & 0.1386 & 0.00 & 0.00 & 424.00 & 90.60 \\
\hline 23 & 24 & 0.0216 & 0.1105 & 0.1872 & 0.00 & 0.00 & 117.00 & 53.10 \\
\hline 11 & 25 & 0.0018 & 0.0227 & 2.1230 & 0.00 & 0.00 & 0.00 & 0.00 \\
\hline 25 & 26 & 0.0019 & 0.0280 & 3.1526 & 0.00 & 0.00 & 0.00 & 0.00 \\
\hline 25 & 27 & 0.0019 & 0.0274 & 3.0741 & 0.00 & 0.00 & 368.00 & 59.60 \\
\hline 23 & 28 & 0.0000 & 0.0062 & 0.0000 & 0.00 & 0.00 & 0.00 & 0.00 \\
\hline 25 & 29 & 0.0014 & 0.0195 & 2.2505 & 0.00 & 0.00 & 174.00 & -8.00 \\
\hline 29 & 30 & 0.0021 & 0.0300 & 3.5289 & 0.00 & 0.00 & 0.00 & 0.00 \\
\hline 40 & 31 & 0.0000 & 0.0062 & 0.0000 & 0.00 & 0.00 & 0.00 & 0.00 \\
\hline 31 & 32 & 0.0022 & 0.0300 & 3.6137 & 0.00 & 0.00 & 0.00 & 0.00 \\
\hline 11 & 33 & 0.0014 & 0.0204 & 2.2869 & 0.00 & 0.00 & 0.00 & 0.00 \\
\hline 35 & 34 & 0.0000 & 0.0590 & 0.0000 & 0.00 & 0.00 & 125.00 & 39.80 \\
\hline 24 & 35 & 0.0180 & 0.0920 & 0.1556 & 0.00 & 0.00 & 0.00 & 0.00 \\
\hline 33 & 36 & 0.0005 & 0.0070 & 0.7856 & 0.00 & 0.00 & 0.00 & 0.00 \\
\hline 38 & 37 & 0.0021 & 0.0107 & 0.0208 & 0.00 & 0.00 & 813.00 & 110.00 \\
\hline 37 & 38 & 0.0021 & 0.0107 & 0.0208 & 0.00 & 0.00 & 612.00 & -455.00 \\
\hline 40 & 39 & 0.0202 & 0.1129 & 0.1993 & 0.00 & 0.00 & 404.00 & 135.00 \\
\hline 37 & 40 & 0.0184 & 0.0949 & 0.0000 & 0.00 & 0.00 & 393.00 & -111.00 \\
\hline 42 & 41 & 0.0106 & 0.0596 & 0.0951 & 0.00 & 0.00 & 262.00 & 13.20 \\
\hline 12 & 42 & 0.0000 & 0.0063 & 0.0000 & 0.00 & 0.00 & 229.00 & 183.00 \\
\hline 41 & 43 & 0.0110 & 0.1184 & 0.2081 & 0.00 & 0.00 & 184.00 & 60.20 \\
\hline 41 & 44 & 0.0229 & 0.1174 & 0.2087 & 0.00 & 0.00 & 139.00 & 53.70 \\
\hline 35 & 45 & 0.0129 & 0.0657 & 0.1155 & 0.00 & 0.00 & 90.10 & 55.30 \\
\hline 32 & 33 & 0.0014 & 0.0195 & 2.2441 & & & & \\
\hline 37 & 38 & 0.0021 & 0.0107 & 0.0208 & & & & \\
\hline 37 & 40 & 0.0184 & 0.0949 & 0.0000 & & & & \\
\hline 37 & 40 & 0.0184 & 0.0949 & 0.0000 & & & & \\
\hline 41 & 42 & 0.0106 & 0.0596 & 0.0951 & & & & \\
\hline 42 & 43 & 0.0172 & 0.0884 & 0.1446 & & & & \\
\hline 42 & 43 & 0.0172 & 0.0884 & 0.1446 & & & & \\
\hline 43 & 44 & 0.0181 & 0.0929 & 0.1671 & & & & \\
\hline 13 & 35 & 0.0096 & 0.0491 & 0.0861 & & & & \\
\hline 13 & 45 & 0.0033 & 0.0167 & 0.3019 & & & & \\
\hline 14 & 37 & 0.0177 & 0.0910 & 0.1510 & & & & \\
\hline 14 & 37 & 0.0177 & 0.0910 & 0.1510 & & & & \\
\hline 14 & 37 & 0.0177 & 0.0910 & 0.1510 & & & & \\
\hline 15 & 16 & 0.0163 & 0.0835 & 0.1411 & & & & \\
\hline 15 & 39 & 0.0250 & 0.1648 & 0.4581 & & & & \\
\hline 17 & 18 & 0.0153 & 0.0861 & 0.1328 & & & & \\
\hline 18 & 44 & 0.0344 & 0.1760 & 0.3061 & & & & \\
\hline 18 & 44 & 0.0344 & 0.1760 & 0.3061 & & & & \\
\hline 19 & 25 & 0.0000 & 0.0300 & 0.0000 & & & & \\
\hline 21 & 22 & 0.0182 & 0.0935 & 0.1602 & & & & \\
\hline 21 & 22 & 0.0182 & 0.0935 & 0.1602 & & & & \\
\hline 21 & 26 & 0.0000 & 0.0062 & 0.0000 & & & & \\
\hline 23 & 24 & 0.0216 & 0.1105 & 0.1872 & & & & \\
\hline 24 & 35 & 0.0180 & 0.0920 & 0.1556 & & & & \\
\hline 25 & 36 & 0.0005 & 0.0070 & 0.7857 & & & & \\
\hline 26 & 27 & 0.0005 & 0.0069 & 0.7728 & & & & \\
\hline 26 & 28 & 0.0012 & 0.0175 & 2.0160 & & & & \\
\hline 30 & 38 & 0.0000 & 0.0062 & 0.0000 & & & & \\
\hline
\end{tabular}


- The performance of the VNS algorithm was compared to a multi-start method. The results showed that the VNS algorithm was more efficient for solving the problem, accrediting it as a powerful tool for the design of controllers and for the analysis of small-signal stability in electric power systems.

- The results presented corroborate the fact that the supplementary damping controllers, with properly adjusted parameters, are able to insert damping to both local and inter-area oscillation modes.

- In particular, it was possible to conclude that the power system stabilizers are capable of inserting damping to the generators' low frequency oscillations, i.e., the local modes, increasing the margin of stability to small-signal disturbances. Moreover, it was shown that the interline power flow controller-power oscillation damping controller operates mainly by inserting damping to the inter-area oscillation modes.

- A robustness analysis demonstrated that the solutions obtained by the VNS algorithm provided high levels of damping even when the operating point of the system changed.

Future work in this subject can be related to including the robustness analysis into the metaheuristic framework to ensure a minimum damping level for a range of operating conditions. Also, advanced stabilizers, such as multi-band, can be considered. It is also possible to extend the formulation to a planning problem, with the objective to find the best places to install the controllers in the system.

\section{Acknowledgment}

This work was supported by the Coordination for the Improvement of Higher Education Personnel (CAPES), the Brazilian National Council for Scientific and Technological Development (CNPq), under grant 141084/2016-2, and the São Paulo Research Foundation (FAPESP), under grant 2014/23741-9.

\section{Appendix A}

Complete data for the Reduced Southern Brazilian system, used in the CSM [3], is provided in Tables 11 and 12. It is assumed that bus 4 is the reference bus. The apparent power base is $100 \mathrm{MVA}$ and the voltage base is $345 \mathrm{kV}$.

\section{References}

[1] Kundur P. Power system stability and control. New York: MacGraw-Hill; 1994.

[2] Larsen E, Swann D. Applying power system stabilizers part II: performance objectives and tuning concepts. IEEE Trans Power Apparat Syst 1981;PAS-100 (6):3025-33.

[3] Fortes EV, Araujo PB, Macedo LH. Coordinated tuning of the parameters of PI, PSS and POD controllers using a specialized Chu-Beasley's genetic algorithm. Electr Power Syst Res 2016;140:708-21.

[4] De Mello FP, Concordia C. Concepts of synchronous machine stability as affected by excitation control. IEEE Trans Power Apparat Syst 1969;88 (4):316-29.

[5] Noroozian M, Andersson G. Damping of power system oscillations by use of controllable components. IEEE Trans Power Deliv 1994;9(4):2046-54.
[6] Valle DB, Araujo PB. The influence of GUPFC FACTS device on small signal stability of the electrical power systems. Int J Electr Power Energy Syst 2015;65:299-306.

[7] Gyugyi L, Sen K, Schauderr CD. The interline power flow controller concept: a new approach to power flow management in transmission systems. IEEE Trans Power Deliv 1999;14(3):1115-23.

[8] Yang N, Liu Q McClley JD. TCSC controller design for damping interarea oscillations. IEEE Trans Power Syst 1998;13(14):1304-10.

[9] Chen CL, Hsu YY. Coordinated synthesis of multimachine power system stabilizer using an efficient decentralized modal control (DMC) algorithm. IEEE Trans Power Syst 1987;2(3):543-50. http://dx.doi.org/10.1109/TPWRS.1987. 4335165.

[10] Araujo PB, Zaneta LC. Pole placement method using the system matrix transfer function and sparsity. Int J Electr Power Syst Energy Syst 2001;23(3):173-8.

[11] Abd-Elazim SM, Ali ES. Power system stability enhancement via bacteria foraging optimization algorithm. Arab J Sci Eng 2013;38(3):599-611.

[12] Abd-Elazim SM, Ali ES. Coordinated design of PSSs and SVC via bacteria foraging optimization algorithm in a multimachine power system. Int J Electr Power Energy Syst 2012;41(1):44-53.

[13] Mondal D, Chakrabarti A, Sengupta A. Optimal placement and parameter setting of SVC and TCSC using PSO to mitigate small signal stability problem. Int J Electr Power Energy Syst 2012;42(1):334-40.

[14] Hasanvand H, Arvan MR, Mozafari B, Amraee T. Coordinated design of PSS and TCSC to mitigate interarea oscillations. Int J Electr Power Energy Syst 2016;78:194-206.

[15] Shayeghi H, Safari A, Shayanfar HA. PSS and TCSC damping controller coordinated design using PSO in multi-machine power system. Energy Convers Manage 2010;51(12):2930-7.

[16] Hassan LH, Moghavvemi M, Almurib HAF, Muttaqi KM. A coordinated design of PSSs and UPFC-based stabilizer using genetic algorithm. IEEE Trans Ind Appl 2014;50(5):2957-66.

[17] Jolfaei MG, Sharaf AM, Shariatmadar SM, Poudeh MB. A hybrid PSS-SSSC GAstabilization scheme for damping power system small signal oscillations. Int J Electr Power Energy Syst 2016;75:337-44.

[18] Bian XY, Geng Y, Lo KL, Fu Y, Zhou QB. Coordination of PSSs and SVC damping controller to improve probabilistic small-signal stability of power system with wind farm integration. IEEE Trans Power Syst 2016;31(3):2371-82.

[19] Gholipour E, Nosratabadi SM. A new coordination strategy of SSSC and PSS controllers in power system using SOA algorithm based on pareto method. Int J Electr Power Energy Syst 2015;67:462-71.

[20] Abd-Elazim SM, Ali ES. Optimal power system stabilizers design via cuckoo search algorithm. Int J Electr Power Energy Syst 2016;75:99-107.

[21] Fortes EV, Araujo PB, Macedo LH, Gamino BR, Martins LFB. Analysis of the influence of PSS and IPFC-POD controllers in small-signal stability using a simulated annealing algorithm. In: 2016 12th IEEE international conference on industry applications (INDUSCON); 2016. p. 1-8.

[22] Panda S, Yegireddy NK, Mohapatra SK. Hybrid BFOA-PSO approach for coordinated design of PSS and SSSC-based controller considering time delays. Int J Electr Power Energy Syst 2013;49:221-33.

[23] Abd-Elazim SM, Ali ES. Synergy of particle swarm optimization and bacterial foraging for TCSC damping controller design. WSEAS Trans Power Syst 2013;8 (2):74-84

[24] Khadanga RK, Satapathy JK. Time delay approach for PSS and SSSC based coordinated controller design using hybrid PSO-GSA algorithm. Int J Electr Power Energy Syst 2015;71:262-73.

[25] Hansen P, Mladenović N. Variable neighborhood search: principles and applications. Eur J Oper Res 2001;130(3):449-67.

[26] Glover F, Kochenberger G. Handbook of metaheuristics. Norwell MA, USA: Kluwer Academic; 2003.

[27] Zhang X-P. Modelling of the interline power flow controller and the generalised unified power flow controller in Newton power flow. IEE ProcGener, Transm Distrib 2003;150(3):268-74.

[28] Zhang J, Yokoyama A. Power system transient stability improvement by the interline power flow controller (IPFC). IEEJ Trans Power Energy 2008;128 (1):208-15.

[29] Fortes EV. Application of the specialized Chu-Beasley's genetic algorithm for adjusting the parameters of supplementary controllers and the IPFC FACTS device [Ph.D. Thesis]. Ilha Solteira: School of Natural Sciences and Engineering, Sao Paulo State University; 2016. p. 73-77 [in Portuguese].

[30] Bazaraa MS, Sherali HD, Shetty CM. Nonlinear programming: theory and algorithms. 3rd ed. Wiley-Interscience; 2006. 